\title{
Kinematics Analysis and Singularities of Novel Decoupled Parallel Manipulators with Simplified Architecture
}

\author{
Raffaele Di Gregorio \\ Department of Engineering, University of Ferrara \\ Via Saragat, 1; 44122 FERRARA; Italy \\ T: +39-0532-974828, F: +39-0532-974870 \\ E: raffaele.digregorio@unife.it
}

\begin{abstract}
Parallel manipulators (PMs) with decoupled kinematics can be obtained by combining a translational PM (TPM) with a spherical PM (SPM) either in multiplatform architectures or in integrated more-complex architectures. Some of the latter type are inspired by the 6-4 fully parallel manipulator (6-4 FPM), whereas others of the same type are deduced by suitably combining TPMs' limbs and SPMs' limbs into more cumbersome limbs which contain more than one actuated joint. The decoupled PMs (DPMs) presented here pursue an intermediate concept between the last two which keeps all the actuators on or near to the base in a simplified architecture with only three limbs. These features preserve the lightness of the mobile masses, together with the associated good dynamic performances, and reduce the limitations on the workspace due to the eliminated limbs and to possible limb interferences. The finite and instantaneous kinematics of the proposed DPMs is studied, thus proving the practical implementation of the proposal.
\end{abstract}

Keywords: parallel manipulators, decoupled kinematics, mobility analysis, singularity locus

\section{Introduction}

The possibility of decoupling position and orientation in end effector's pose control is an appealing feature. It allows the use of simplified path-planning algorithms which sequentially involve a limited number of actuators and make the machine behave in an easy-to-visualize manner that facilitates the operator. This decoupling is easy to implement in serial manipulators [1], whereas it requires a specific design in parallel manipulators (PMs) $[2-23]$ due to their intrinsically coupled kinematics.

Four-degrees-of-freedom(4-dof) PMs with decoupled input-output relationships have been proposed [12 - 14$]$ which feature position/orientation decoupling, too. Also, the 4-dof (quadrupteron [15, 16]) and 5-dof (pentapteron $[17,18])$ PMs of the multipteron family [18] deserve to be mentioned among the lower-mobility PMs with decoupled position and orientation. These multipteron PMs have a number of limbs equal to their dof number and only one actuated pair per limb which is a prismatic pair adjacent to the base. They are a clear proof 
that putting the actuators on (or near) the base is a key requirement to get PMs with high dynamic performances $[18]$

Six-dof PMs with decoupled kinematics can be obtained by combining in series a translational PM (TPM) and a spherical PM (SPM) into a multiplatform architecture [5]. Such simple solutions, in general, lose many of the advantages of parallel architectures. Combining a TPM and an SPM so that the TPM controls the position of the spherical motion center of the SPM $[6,20]$ is a better idea whose most known implementation is the 6-4 fully parallel manipulator (6-4 FPM) [2]. This concept generates many decoupled PM (DPM) architectures since many are the TPM and SPM architectures proposed in the literature [24]. The so-deduced DPM architectures feature six limbs with one actuator per limb (three of the TPM and three of the SPM) which still is somehow cumbersome.

An alternative concept relies on the identification of suitable limb types which give the sought-after decoupling when put together $[3,10,11,20,22,23]$. This technique has the advantage of identifying architectures with a reduced number of limbs (usually three [11,20,23]), but requires more than one actuator per limb.

Here, a family of 63 DPM architectures with three limbs is identified and, then, studied through a unified approach. Such architectures feature all the actuators on or near to the base. This property makes them save about all the advantages of 6-dof PMs with six limbs and one actuator per limb. Also, their three-limbed architecture makes their workspace wider since the occurrence of limb interferences and the constraints on the workspace [25] due to the limbs are reduced; whereas, their decoupled position analysis makes their control easier.

This paper is organized as follows. Section 2 presents the novel DPM architectures. Sections 3 and 4 address their position analysis and their instantaneous kinematics, respectively. Section 5 applies the deduced formulas to give some hints on how could be workspace, statics behavior, positioning precision and isotropy/stiffness of the identified architectures. Eventually, section 6 draws the conclusions.

\section{Type Synthesis}

Parallel manipulators (PMs) feature two rigid bodies, one fixed (base) and the other mobile (platform) connected to one another through a number of kinematic chains (limbs). Hereafter, the notation $\mathrm{g}_{1} \mathrm{string}_{1}-\mathrm{g}_{2} \mathrm{string}_{2}-\ldots-$ $\mathrm{g}_{\mathrm{n}}$ string $\mathrm{n}_{\mathrm{n}}$, where $\mathrm{g}_{\mathrm{i}}$ for $\mathrm{i}=1, \ldots, \mathrm{n}$ are integers, denotes a structure or a manipulator constituted by two rigid bodies, named base and platform, joined through $\left(g_{1}+g_{2}+\ldots+g_{n}\right)$ kinematic chains (limbs) whose topologies are given by the strings separated with hyphens. The i-th string, string ${ }_{i}$, lists the sequence of kinematic pairs encountered when moving from the base to the platform and the number $g_{i}$ indicates how many limbs with that topology are 
present; $\mathrm{g}_{\mathrm{i}}$ is omitted if it is equal to one. Capital letters indicate the type of kinematic pair in these strings: $\mathrm{S}, \mathrm{P}$, $\mathrm{R}$ and $\mathrm{U}$ stand for spherical, prismatic, revolute pairs and universal joint, respectively, and an underlined capital letter indicates an actuated pair when the notation refers to manipulators' architectures; also, the symbols $\|$ or $\perp$ between two $\mathrm{R}$ indicate that the axes of the two adjacent $\mathrm{R}$ pairs are parallel or perpendicular to one another, respectively. Eventually, the phrase "limb connectivity" indicates the degrees of freedom of the relative motion between platform and base in the kinematic chain constituted by platform and base uniquely connected through the limb whose connectivity [26-28] is considered.

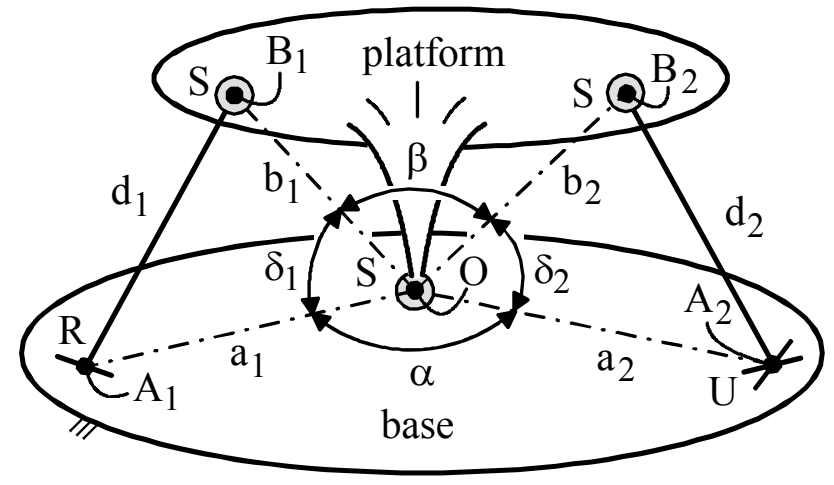

Figure 1: A structure of S-RS-US type

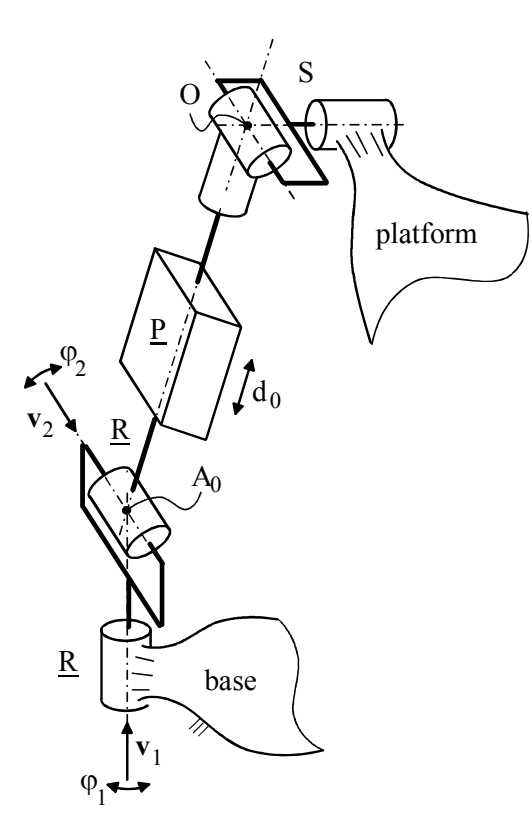

(a)

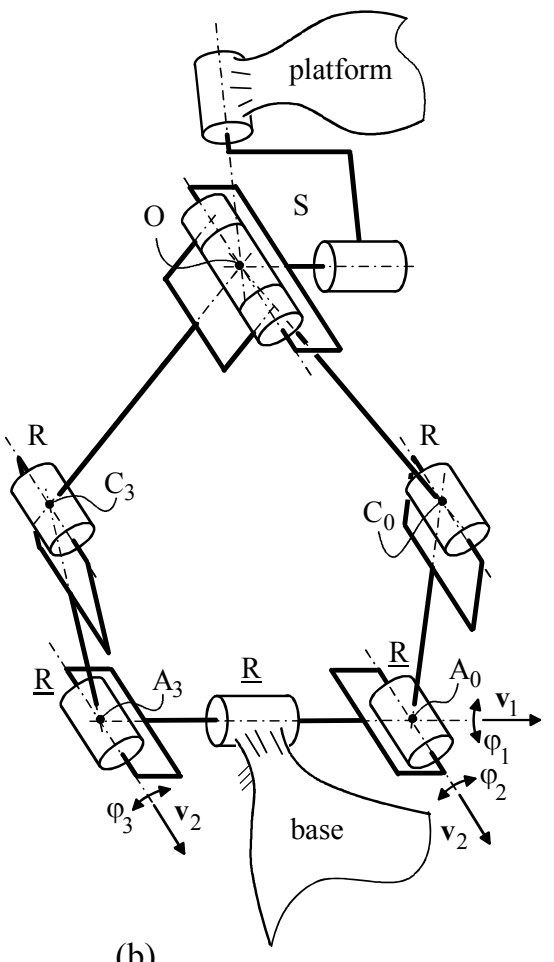

(b)

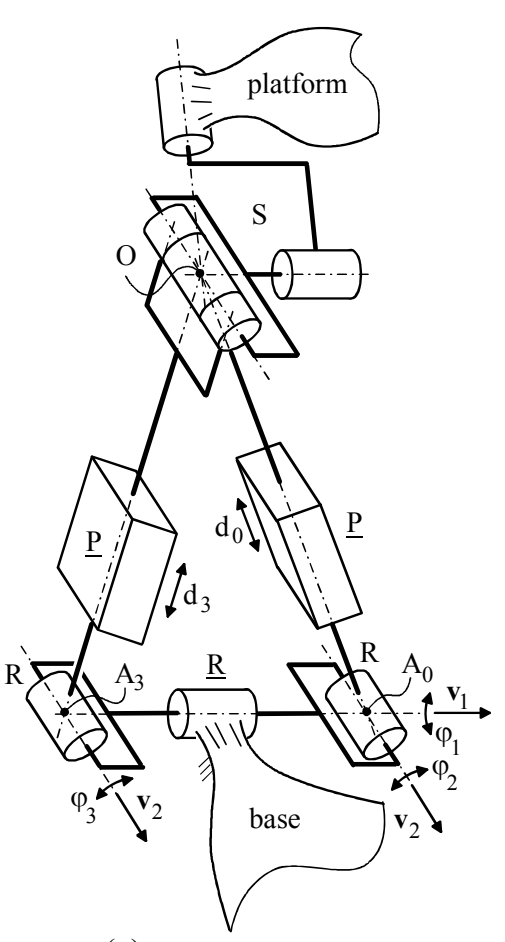

(c)

Figure 2: Three limbs of ( $3 \mathrm{~W}) \mathrm{S}$ type: (a) the $\underline{\mathrm{R} R P} \mathrm{~S}$ limb, (b) the single-loop $\underline{\mathrm{R}} \perp(2 \underline{\mathrm{R}} \mid \mathrm{R}) \mathrm{S}$ limb, and (c) singleloop $\underline{\mathrm{R}} \perp(2 \mathrm{R} \underline{\mathrm{P}}) \mathrm{S}$ limb. 
Figure 1 shows an S-RS-US structure. With reference to Fig. 1, $\mathrm{O}$ is the center of the $\mathrm{S}$ pair that directly joins platform and base. $B_{1}$ and $A_{1}$ are the S-pair center and the foot of the perpendicular passing through $B_{1}$ on the revolute-pair axis, respectively, in the RS limb; whereas, $A_{2}$ and $B_{2}$ are the centers of the $U$ joint and of the $S$ pair, respectively, in the US limb.

The proposed family of DPMs is constituted by all the three-limbed architectures with actuators on or near to the base that generate an S-RS-US structure (Fig. 1) when the actuators are locked. The deduction of these architectures moves from the fact that "each limb of a six-dof PM has to guarantee six degrees of freedom to the relative motion between platform and base (i.e., the limb connectivity must be six)". Henceforth, only six-dof limbs (i.e., non-redundant limbs) will be considered. This assumption and the previous consideration bring to state that, in the sought-after architectures,

(i) the S limb of the S-RS-US structure must be generated by a limb of $(\underline{3 \mathrm{~W}}) \mathrm{S}$ type where the platform is joined through an S pair to one link of a spatial three-dof mechanism, generically denoted $(\underline{3 \mathrm{~W}})$, with three actuated pairs,

(ii) the RS limb of the S-RS-US structure must be generated by a limb either of $\underline{X R Y S}$ type or of XYRS type or of R $\underline{X Y S}$ type where $\mathrm{X}$ and $\mathrm{Y}$ are any two single-dof pairs/mechanisms, and

(iii) the US limb of the S-RS-US structure must be generated by a limb either of $\underline{Z} U S$ type or of UZS type where $\mathrm{Z}$ is a single-dof pair/mechanism.

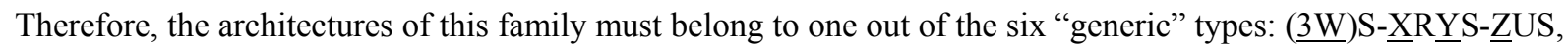

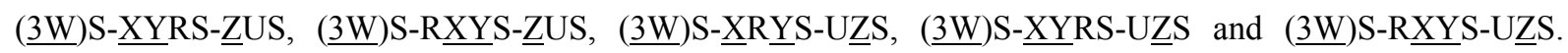
Each particular architecture is fully defined when the topologies of the $(\underline{3 \mathrm{~W}}), \underline{X}, \underline{\mathrm{Y}}$ and $\underline{\mathrm{Z}}$ pairs/mechanisms are defined. These topologies are particularized by taking into account the other two structural conditions that identify the family, that is, (a) the manipulator must be a DPM and (b) the actuated pairs must be on or near to the base. An actuated P pair can be considered "near to the base" even though it is adjacent to the S pair that joins the limb to the platform since U $\underline{P S}$ or S $\underline{P S}$ limbs are commonly adopted in fully-parallel manipulators [29]; whereas, an actuated R pair can be considered "near to the base" if it is adjacent to another R pair that joins the limb to the base since, in this case, it can be easily actuated by keeping the actuator on the base.

The generic $(\underline{3 \mathrm{~W}}) \mathrm{S}$ limb is sufficient to satisfy condition (a) since the spatial 3-dof mechanism, $(\underline{3 \mathrm{~W}})$, is able to fully control the position of one platform point (point $\mathrm{O}$ of Fig. 1) with its three actuated pairs no matter how the other two limbs are devised. The topology of the $(\underline{3 \mathrm{~W}})$ mechanism is limited by condition (b) and the fact that it has to constitute a unique limb when combined with the S pair. $(\underline{3 \mathrm{~W}}) \mathrm{S}$ limbs that satisfy these constraints are the $\underline{R R P S} \operatorname{limb}$ (Fig. 2(a)) and either of the two single-loop $\underline{\mathrm{R}} \perp(2 \underline{\mathrm{R}} \| \mathrm{R}) \mathrm{S}$ and $\underline{\mathrm{R}} \perp(2 \mathrm{R} \underline{\mathrm{P}}) \mathrm{S}$ limbs shown in Figs. 2(b) 
and $2(\mathrm{c})$, respectively. The $(\underline{3 \mathrm{~W}})$ mechanisms of the $\underline{\mathrm{R}} \perp(2 \underline{\mathrm{R}} \| \mathrm{R}) \mathrm{S}$ and $\underline{\mathrm{R}} \perp(2 \mathrm{R} \underline{\mathrm{P}}) \mathrm{S}$ limbs are both five-bar planar linkages whose motion plane is hinged to the base through an actuated R pair. Even though other solutions (e.g.,

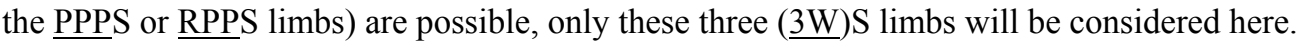

Regarding the remaining two limbs, if $\underline{X}, \underline{Y}$ and $\underline{Z}$ are either $\underline{\mathrm{R}}$ or $\underline{\mathrm{P}}$ pairs and the limbs that do not satisfy condition (b) are excluded, the following limb types are identified:

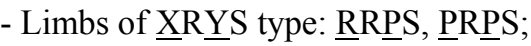

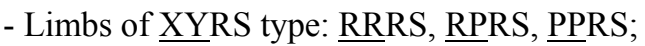

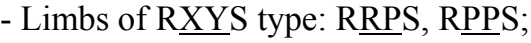

- Limbs of ZUUS type: RUS, $\underline{\text { PUS; }}$

- Limbs of UZS type: UPSS.

The combination of the above-identified limb types yields $63(=3 \times 7 \times 3)$ DPM architectures. One out of these DPM architectures is the RRPS-RRPS-UPS (Fig. 3) which deserves to be highlighted for its symmetry since it features three limbs with the same RRPS topology that are actuated in different ways.

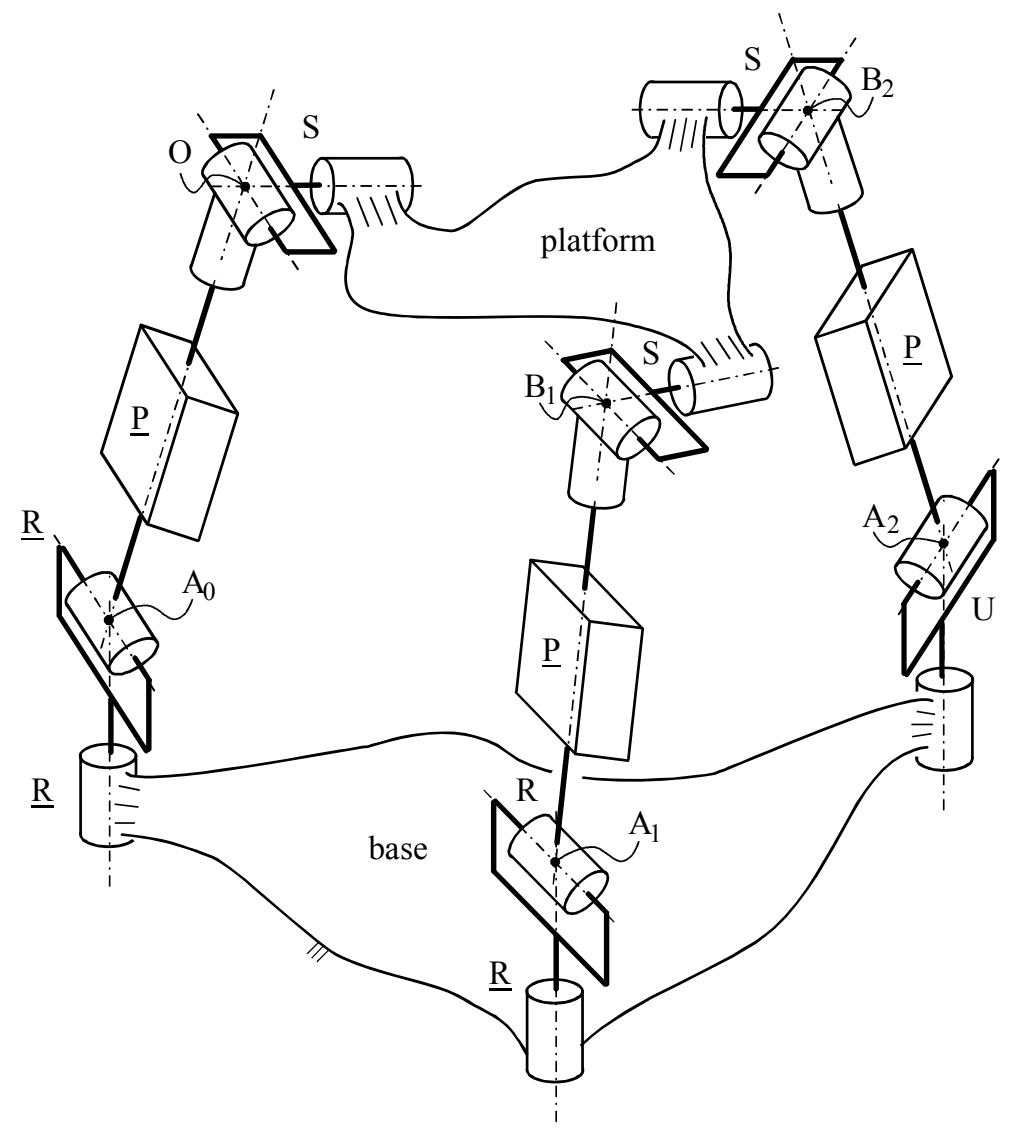

Figure 3: The $\underline{R R P S}-\underline{R R} \underline{P} S-U \underline{P S}$ architecture. 


\section{Position Analysis}

PMs' position analysis refers to the solution of two finite-kinematics problems: the direct position analysis (DPA) and the inverse position analysis (IPA). The DPA is the determination of the platform poses that are compatible with assigned values of the actuated-joint variables; vice versa, the IPA is the determination of the values of the actuated-joint variables that are compatible with an assigned platform pose.

The DPA of all the above-identified DPM architectures can be solved with the same algorithm since their DPA solutions one-to-one correspond to the assembly modes of the S-RS-US structure generated when the actuators are locked. Instead, their IPA depends on the adopted limb types. Actually, the assigned platform pose gives the positions of the three S-pair centers (i.e., points $\mathrm{O}, \mathrm{B}_{1}$ and $\mathrm{B}_{2}$ in Fig. 1) and the IPA of each limb yields the limb configurations compatible with that pose. Thus, DPM's IPA solutions correspond to the combinations of all the configurations the three limbs can assume for one assigned position of their ending S-pair center.

\subsection{Direct Position Analysis:}

An algorithm that determines the assembly modes of a general S-RS-US structure (Fig. 1) is presented in this subsection. The sketch of Fig 4 extracts all the geometric constants of the S-RS-US structure shown in Fig. 1 and defines the notations that will be adopted.

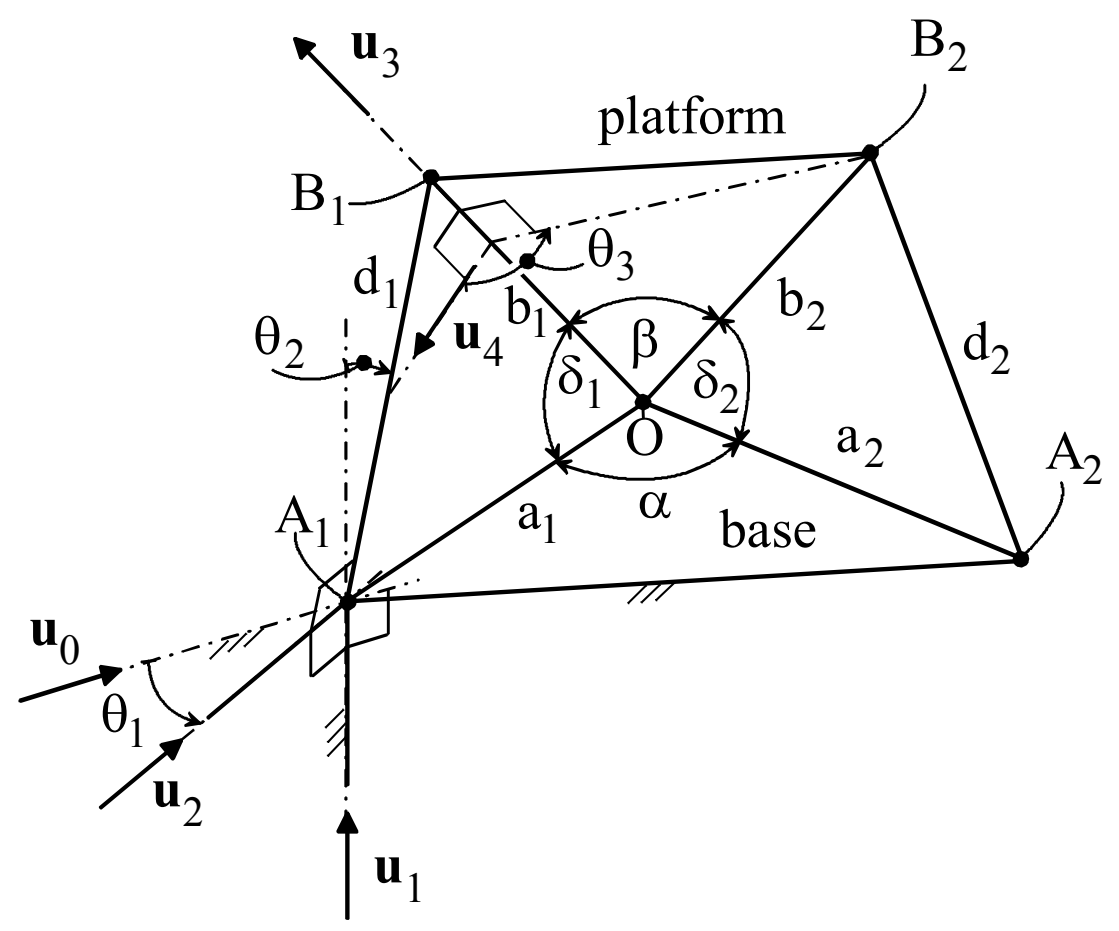

Figure 4: S-RS-US structure: notations. 
With reference to Figs. 1 and 4, the triangle $\mathrm{B}_{1} \mathrm{OB}_{2}$ defines the platform geometry, that is, the angle $\beta$ and the lengths $b_{1}$ and $b_{2}$ of the segments $\mathrm{OB}_{1}$ and $\mathrm{OB}_{2}$, respectively, are geometric constants of the platform. The triangle $\mathrm{A}_{1} \mathrm{OA}_{2}$ has an assigned geometry and is fixed to the base once the actuated-joint variables of the DPM that generated this S-RS-US structure are assigned, that is, the angle $\alpha$ and the lengths $a_{1}$ and $a_{2}$ of the segments $\mathrm{OA}_{1}$ and $\mathrm{OA}_{2}$, respectively, depend on the actuated-joint variables. $\mathbf{u}_{\mathrm{i}}$ for $\mathrm{i}=0,1, \ldots, 4$ are unit vectors: $\mathbf{u}_{2}$ is parallel to the direction of the R-pair axis of the RS limb, $\mathbf{u}_{0}$ and $\mathbf{u}_{1}$ are parallel to two mutually orthogonal directions fixed to the base with $\mathbf{u}_{1}$ perpendicular to $\mathbf{u}_{2}$ too; also, $\mathbf{u}_{3}$ is parallel to the segment $\mathrm{OB}_{1}$, which is fixed to the platform, and $\mathbf{u}_{4}$ is perpendicular to the segment $\mathrm{OB}_{1}$ and lies on the triangle $\mathrm{B}_{1} \mathrm{OA}_{1}$. $\mathrm{d}_{1}$ and $\mathrm{d}_{2}$ are the lengths of the segments $A_{1} B_{1}$ and $A_{2} B_{2}$, respectively. The angle $\theta_{1}$ is a geometric constant of the RS limb that depends on the actuated-joint variables of the kinematic chain that generated it; whereas, the angle $\theta_{2}$ is the joint variable of the R pair of the RS limb; eventually, $\theta_{3}$ is an angle whose value, together with the abovedefined geometric parameters, uniquely identifies the assembly mode of the S-RS-US structure. In the $\underline{\text { RRPS- }}$ $\underline{\text { RRPS-UPS }}$ architecture (Fig. 3), $\mathrm{d}_{1}$ and $\mathrm{d}_{2}$ are actuated-joint variables and, if $\mathbf{u}_{1}$ is chosen parallel the axis of the actuated $\mathrm{R}$ pair of the $\underline{\mathrm{R}} \underline{\mathrm{P}} \mathrm{S} \operatorname{limb}, \theta_{1}$ is an actuated-joint variable, too.

The following analytic relationships hold among the above-defined geometric parameters (all the vectors are measured in a reference system embedded in the base):

$$
\begin{aligned}
& \mathbf{u}_{2}=\mathbf{u}_{0} \cos \theta_{1}+\left(\mathbf{u}_{1} \times \mathbf{u}_{0}\right) \sin \theta_{1}, \quad \mathbf{u}_{2} \times \mathbf{u}_{1}=\left(\mathbf{u}_{0} \times \mathbf{u}_{1}\right) \cos \theta_{1}+\mathbf{u}_{0} \sin \theta_{1} \\
& \left(\mathbf{B}_{1}-\mathbf{A}_{1}\right)=\mathrm{d}_{1}\left[\mathbf{u}_{1} \cos \theta_{2}+\left(\mathbf{u}_{2} \times \mathbf{u}_{1}\right) \sin \theta_{2}\right], \quad\left(\mathbf{B}_{1}-\mathbf{O}\right)=\left(\mathbf{B}_{1}-\mathbf{A}_{1}\right)+\left(\mathbf{A}_{1}-\mathbf{O}\right) \\
& \mathbf{u}_{3}=\frac{\left(\mathbf{B}_{1}-\mathbf{O}\right)}{\mathrm{b}_{1}} \equiv \frac{\mathrm{d}_{1}\left[\mathbf{u}_{1} \cos \theta_{2}+\left(\mathbf{u}_{2} \times \mathbf{u}_{1}\right) \sin \theta_{2}\right]+\left(\mathbf{A}_{1}-\mathbf{O}\right)}{\mathrm{b}_{1}} \\
& \sin \delta_{\mathrm{j}}=\sqrt{1-\left(\frac{\mathrm{b}_{\mathrm{j}}^{2}+\mathrm{a}_{\mathrm{j}}^{2}-\mathrm{d}_{\mathrm{j}}^{2}}{2 \mathrm{a}_{\mathrm{j}} \mathrm{b}_{\mathrm{j}}}\right)^{2}, \quad \mathrm{j}=1,2} \\
& \mathbf{u}_{4}=\frac{\left(\mathbf{A}_{1}-\mathbf{O}\right)-\mathbf{u}_{3}\left[\left(\mathbf{A}_{1}-\mathbf{O}\right) \cdot \mathbf{u}_{3}\right]}{\mathrm{a}_{1} \sin \delta_{1}} \\
& \mathbf{u}_{3} \times \mathbf{u}_{4}=\frac{\mathbf{u}_{3} \times\left(\mathbf{A}_{1}-\mathbf{O}\right)}{\mathrm{a}_{1} \sin \delta_{1}} \equiv \frac{\mathrm{d}_{1}\left[\mathbf{u}_{1} \cos \theta_{2}+\left(\mathbf{u}_{2} \times \mathbf{u}_{1}\right) \sin \theta_{2}\right] \times\left(\mathbf{A}_{1}-\mathbf{O}\right)}{\mathrm{a}_{1} \mathrm{~b}_{1} \sin \delta_{1}} \\
& \left(\mathbf{B}_{2}-\mathbf{O}\right)=\left(\mathbf{B}_{2}-\mathbf{A}_{2}\right)+\left(\mathbf{A}_{2}-\mathbf{O}\right) \equiv \mathrm{b}_{2}\left\{\mathbf{u}_{3} \cos \beta+\sin \beta\left[\mathbf{u}_{4} \cos \theta_{3}+\left(\mathbf{u}_{3} \times \mathbf{u}_{4}\right) \sin \theta_{3}\right]\right\}
\end{aligned}
$$

where $\mathbf{u}_{0}, \mathbf{u}_{1},\left(\mathbf{A}_{1}-\mathbf{O}\right)$ and $\left(\mathbf{A}_{2}-\mathbf{O}\right)$ are known constant vectors and $\theta_{1}$ is a known constant angle.

Relationships (1) when introduced into the closure equations (see Fig. 4) 
$\mathrm{b}_{1}{ }^{2}=\left(\mathbf{B}_{1}-\mathbf{O}\right) \cdot\left(\mathbf{B}_{1}-\mathbf{O}\right) \equiv\left[\left(\mathbf{B}_{1}-\mathbf{A}_{1}\right)+\left(\mathbf{A}_{1}-\mathbf{O}\right)\right] \cdot\left[\left(\mathbf{B}_{1}-\mathbf{A}_{1}\right)+\left(\mathbf{A}_{1}-\mathbf{O}\right)\right]$

$\mathrm{d}_{2}{ }^{2}=\left(\mathbf{B}_{2}-\mathbf{A}_{2}\right) \cdot\left(\mathbf{B}_{2}-\mathbf{A}_{2}\right) \equiv\left[\left(\mathbf{B}_{2}-\mathbf{O}\right)-\left(\mathbf{A}_{2}-\mathbf{O}\right)\right] \cdot\left[\left(\mathbf{B}_{2}-\mathbf{O}\right)-\left(\mathbf{A}_{2}-\mathbf{O}\right)\right]$

yield the following two equations in explicit form

$\mathrm{a}_{1}^{2}-\mathrm{b}_{1}^{2}+\mathrm{d}_{1}^{2}+2 \mathrm{~d}_{1}\left(\mathbf{A}_{1}-\mathbf{O}\right) \cdot\left[\mathbf{u}_{1} \cos \theta_{2}+\left(\mathbf{u}_{2} \times \mathbf{u}_{1}\right) \sin \theta_{2}\right]=0$

$\mathrm{a}_{2}{ }^{2}+\mathrm{b}_{2}{ }^{2}-\mathrm{d}_{2}{ }^{2}-2 \mathrm{~b}_{2}\left(\mathbf{A}_{2}-\mathbf{O}\right) \cdot\left\{\mathbf{u}_{3} \cos \beta+\sin \beta\left[\mathbf{u}_{4} \cos \theta_{3}+\left(\mathbf{u}_{3} \times \mathbf{u}_{4}\right) \sin \theta_{3}\right]\right\}=0$

Equations (3) constitute a system of two trigonometric equations in the two unknowns $\theta_{2}$ and $\theta_{3}$. Actually, Eq. (3a) contains only $\theta_{2}$; thus, it can be immediately solved by transforming it into a quadratic equation in $\tan \left(\theta_{2} / 2\right)$ through the two trigonometric identities $\cos \theta_{2}=\left[1-\tan ^{2}\left(\theta_{2} / 2\right)\right] /\left[1+\tan ^{2}\left(\theta_{2} / 2\right)\right]$ and $\left.\sin \theta_{2}=2 \tan \left(\theta_{2} / 2\right)\right] /\left[1+\tan ^{2}\left(\theta_{2} / 2\right)\right]$ (see Appendix A). Such equation gives at most two values for $\theta_{2}$ that geometrically correspond to the two possible intersections (see Fig. 4) between the sphere with center $\mathrm{O}$ and radius $\mathrm{b}_{1}$ and the circumference with center $A_{1}$ and radius $d_{1}$ that lies on a plane perpendicular to $\mathbf{u}_{2}$.

If the value of $\theta_{2}$ is known, Eq. (3b) becomes a trigonometric equation in $\theta_{3}$ which can be immediately solved by transforming it into a quadratic equation in $\tan \left(\theta_{3} / 2\right)$ through the two trigonometric identities $\cos \theta_{3}=[1-$ $\left.\tan ^{2}\left(\theta_{3} / 2\right)\right] /\left[1+\tan ^{2}\left(\theta_{3} / 2\right)\right]$ and $\left.\sin \theta_{3}=2 \tan \left(\theta_{3} / 2\right)\right] /\left[1+\tan ^{2}\left(\theta_{3} / 2\right)\right]$ (see Appendix A). Therefore, at most two values of $\theta_{3}$ can be computed through Eq. (3b) for each value of $\theta_{2}$ computed through Eq. (3a). These two values of $\theta_{3}$ geometrically correspond to the two possible intersections between the sphere with center $\mathrm{A}_{2}$ and radius $\mathrm{d}_{2}$ and the circumference with center on $\mathrm{OB}_{1}$ and radius $\mathrm{b}_{2} \sin \beta$ that lies on a plane perpendicular to $\mathbf{u}_{3}$.

The conclusion is that there are at most four assembly modes of a general S-RS-US structure and as many are the DPA solutions of all the studied DPMs.

\subsection{Inverse Position Analysis:}

Here, the positions of the three spherical-pair centers (i.e., points $\mathrm{O}, \mathrm{B}_{1}$ and $\mathrm{B}_{2}$ in Fig. 3) are known since the platform pose is assigned and the values of the actuated-joint variables must be computed. Each of these centers is the ending point of a limb and its position is the input datum of this limb's IPA. Thus, each limb's IPA can be solved independently from the others and, then, the IPA solutions of the analyzed DPM are obtained by combining the IPA solutions of each limb. 
The IPAs of all the serial 6-dof kinematic chains (limbs) with an ending S pair and containing only R and/or P pairs have been solved in closed form in [1] where the demonstration that at most four limb configurations are compatible with an assigned position of the S-pair center is provided. Moreover, in [30] (as reported in [31], pp. 160-161), Takano showed that, with a general layout, (i) at most four solutions are found for limbs containing either three R pairs, or two R pairs and one P pair, whereas (ii) at most two solutions are found for limbs containing one R pair and two P pairs. Condition (i) occurs for four (i.e., $\underline{R} R \underline{P S}, \underline{R R R S}, \underline{R P R S}, \underline{R P S})$ out of the above-listed seven kinematic-chain types that generate an RS limb, when the actuators are locked, and condition (ii) occurs for the remaining three (i.e., $\underline{P R} \underline{P S}, \underline{P P R S}, \underline{R P S})$.

The number of IPA solutions can be further reduced if the limbs satisfy special geometric conditions. This is the case of the three types of kinematic chain which generate a US limb when the actuators are locked. In particular, the UPS limb yields only one solution for the distance $\mathrm{d}_{2}$ (i.e., the only actuated-joint variable of this limb) between $A_{2}$ and $B_{2}$ (see Fig. 4) since $A_{2}$ is a fixed point of the base. Instead, the $\underline{R} U S$ and $\underline{P} U S$ limbs yield two solutions for the actuated-joint variable. In fact, in these two cases, $\mathrm{d}_{2}$ is a fixed distance, and $\mathrm{A}_{2}$ must simultaneously lie on the sphere with center $B_{2}$ and radius $d_{2}$ and on either a circumference, in the case of the RUS limb, or a line, in the case of the PUS limb, both fixed to the base; thus, $A_{2}$ may be located at either of the at-most-two intersections between a sphere and a circumference or a line.

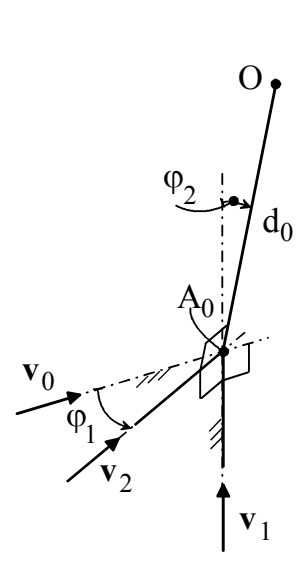

(a)

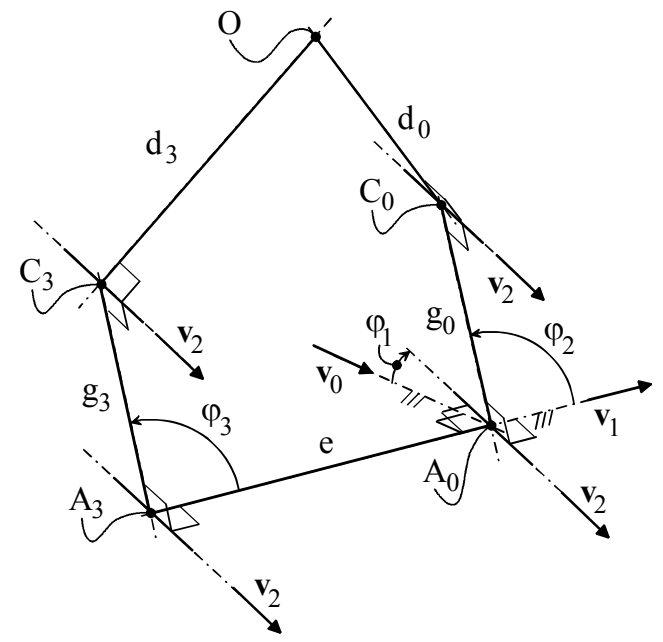

(b)

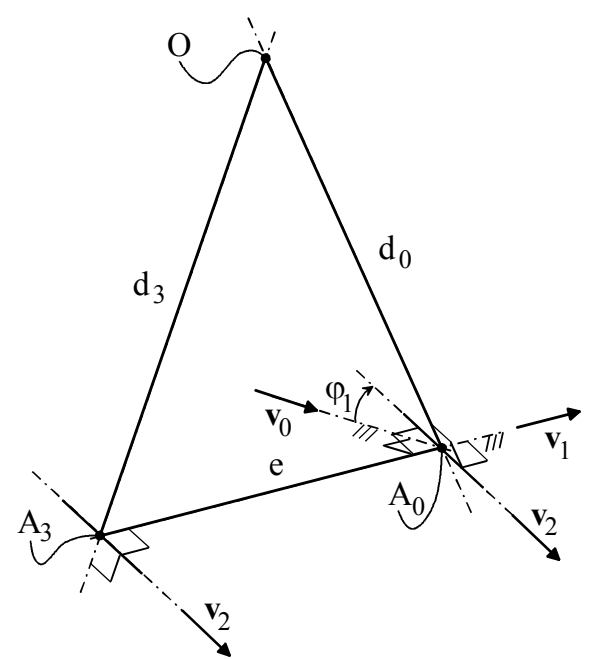

(c)

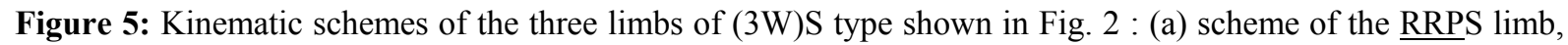
(b) scheme of the single-loop $\underline{\mathrm{R}} \perp(2 \underline{\mathrm{R}} \| \mathrm{R}) \mathrm{S}$ limb, and (c) scheme of the single-loop $\underline{\mathrm{R}} \perp(2 \mathrm{R} \underline{\mathrm{P}}) \mathrm{S}$ limb.

The same holds for the RRPS limb of Fig. 2a whose kinematic scheme is reported in Fig.5a where $\mathbf{v}_{1}$ and $\mathbf{v}_{2}$ are the unit vectors of the axes of the two actuated $\mathrm{R}$ pairs, and $\mathbf{v}_{0}$ is a unit vector fixed to the base and 
perpendicular to $\mathbf{v}_{1}$. With reference to Fig. $5 \mathrm{a}$, the actuated-joint variables are $\varphi_{1}, \varphi_{2}$ and $\mathrm{d}_{0} ; \mathrm{d}_{0}$ is uniquely determined since it is the length of the segment $\mathrm{A}_{0} \mathrm{O}$ and $\mathrm{A}_{0}$ is a fixed point of the base; moreover, the relationship (see Fig. 5a)

$\mathbf{v}_{2}=\mathbf{v}_{0} \cos \varphi_{1}+\left(\mathbf{v}_{1} \times \mathbf{v}_{0}\right) \sin \varphi_{1} \equiv \frac{\mathbf{v}_{1} \times\left(\mathbf{O}-\mathbf{A}_{0}\right)}{\left|\mathbf{v}_{1} \times\left(\mathbf{O}-\mathbf{A}_{0}\right)\right|}$

makes it possible to uniquely determine $\varphi_{1}$ and $\varphi_{2}$ as follows:

$$
\begin{aligned}
& \varphi_{1}=\operatorname{atan} 2\left(\frac{\mathbf{v}_{1} \times\left(\mathbf{O}-\mathbf{A}_{0}\right)}{\left|\mathbf{v}_{1} \times\left(\mathbf{O}-\mathbf{A}_{0}\right)\right|} \cdot\left(\mathbf{v}_{1} \times \mathbf{v}_{0}\right), \frac{\mathbf{v}_{1} \times\left(\mathbf{O}-\mathbf{A}_{0}\right)}{\left|\mathbf{v}_{1} \times\left(\mathbf{O}-\mathbf{A}_{0}\right)\right|} \cdot \mathbf{v}_{0}\right) \\
& \varphi_{2}=\arcsin \left(\frac{\left|\mathbf{v}_{1} \times\left(\mathbf{O}-\mathbf{A}_{0}\right)\right|}{\mathrm{d}_{0}}\right)
\end{aligned}
$$

where $\varphi_{2} \in[0, \pi] \mathrm{rad}$.

Regarding the two single-loop limbs that have been proposed above to control the platform position [i.e., the $\underline{\mathrm{R}} \perp(2 \underline{\mathrm{R}} \| \mathrm{R}) \mathrm{S} \operatorname{limb}$ (Figs. $2 \mathrm{~b}$ and $5 \mathrm{~b})$ and the $\underline{\mathrm{R}} \perp(2 \mathrm{R} \underline{\mathrm{P}}) \mathrm{S}$ limb (Figs. 2c and 5c)], the actuated-joint variable, $\varphi_{1}$ in Figs. $5 \mathrm{~b}$ and $5 \mathrm{c}$, of the actuated $\mathrm{R}$ pair that joins the two limb types to the base and identifies the plane of the triangle $\mathrm{A}_{0} \mathrm{OA}_{3}$ is uniquely determined when the position of point $\mathrm{O}$ is assigned, since points $\mathrm{A}_{0}$ and $\mathrm{A}_{3}$ are fixed point of the base and the relationship (see Figs. $5 \mathrm{~b}$ and $5 \mathrm{c}$ )

$\mathbf{v}_{2}=\mathbf{v}_{0} \cos \varphi_{1}+\left(\mathbf{v}_{1} \times \mathbf{v}_{0}\right) \sin \varphi_{1} \equiv \frac{\left(\mathbf{A}_{3}-\mathbf{O}\right) \times\left(\mathbf{A}_{0}-\mathbf{O}\right)}{\left|\left(\mathbf{A}_{3}-\mathbf{O}\right) \times\left(\mathbf{A}_{0}-\mathbf{O}\right)\right|}$

makes it possible to uniquely determine $\varphi_{1}$ as follows

$$
\varphi_{1}=\operatorname{atan} 2\left(\frac{\left(\mathbf{A}_{3}-\mathbf{O}\right) \times\left(\mathbf{A}_{0}-\mathbf{O}\right)}{\left|\left(\mathbf{A}_{3}-\mathbf{O}\right) \times\left(\mathbf{A}_{0}-\mathbf{O}\right)\right|} \cdot\left(\mathbf{v}_{1} \times \mathbf{v}_{0}\right), \frac{\left(\mathbf{A}_{3}-\mathbf{O}\right) \times\left(\mathbf{A}_{0}-\mathbf{O}\right)}{\left|\left(\mathbf{A}_{3}-\mathbf{O}\right) \times\left(\mathbf{A}_{0}-\mathbf{O}\right)\right|} \cdot \mathbf{v}_{0}\right)
$$


Moreover, the lengths, $\mathrm{d}_{0}$ and $\mathrm{d}_{3}$, of the segments $\mathrm{A}_{0} \mathrm{O}$ and $\mathrm{A}_{3} \mathrm{O}$ (see Fig. 5c), which are the remaining two actuated-joint variables of the $\underline{R} \perp(2 \mathrm{R} \underline{\mathrm{P}}) \mathrm{S} \operatorname{limb}$ (Fig. 2c), are uniquely determined, too, since points $\mathrm{A}_{0}$ and $\mathrm{A}_{3}$ are fixed point of the base. Thus, the IPA of the $\underline{R} \perp(2 \mathrm{R} \underline{\mathrm{P}}) \mathrm{S}$ limb has only one solution.

Eventually, both the angles, $\varphi_{2}$ and $\varphi_{3}$ of Fig. $5 \mathrm{~b}$, that are the remaining two actuated-joint variables of the $\underline{\mathrm{R}} \perp(2 \underline{\mathrm{R}} \| \mathrm{R}) \mathrm{S}$ limb (Fig. 2b) have a double determination for an assigned position of point $\mathrm{O}$, since each point $\mathrm{C}_{\mathrm{i}}$ for $\mathrm{i}=0,3$ (see Fig. 5b) may be located at either of the two intersections of the two circumferences, one with center $\mathrm{O}$ and radius $d_{i}$ and the other with center $A_{i}$ and radius $g_{i}$, that lie on the plane of the triangle $A_{0} \mathrm{OA}_{3}$. Thus, the IPA of the $\underline{\mathrm{R}} \perp(2 \underline{\mathrm{R}} \| \mathrm{R}) \mathrm{S}$ limb has at most four solutions.

In short, the number of IPA solutions of the above-identified 63 DPM architectures varies from one to thirty-two $(=4 \times 2 \times 4)$ according to the chosen limb types. It is worth stressing that the $\underline{R R P S}-\underline{R} R \underline{P S}-U \underline{P S}$ architecture shown in Fig. 3 has only one IPA solution.

\section{Instantaneous Kinematics}

The instantaneous kinematics of all the proposed DPMs relates the velocity of point $\mathrm{O}, \dot{\mathbf{O}}$, and the angular velocity of the platform, $\omega$, to the joint rates of the six actuated-joint variables. The six actuated-joint rates of the studied DPMs can be separated into three sub-set: the three, $\dot{\mathbf{q}}=\left(\dot{\mathrm{q}}_{1}, \dot{\mathrm{q}}_{2}, \dot{\mathrm{q}}_{3}\right)^{\mathrm{T}}$, of the $(3 \mathrm{~W}) \mathrm{S}$ limb, which control $\dot{\mathbf{O}}$, the two, $\dot{\xi}=(\dot{\mathrm{x}}, \dot{\mathrm{y}})^{\mathrm{T}}$, of the above-introduced $\underline{X}$ and $\underline{Y}$ actuated pairs, which control the geometry of the RS limb, and the remaining one, $\dot{z}$, of the above-introduced $\underline{Z}$ actuated pair, which controls the geometry of the US limb.

Regarding the velocity of point $\mathrm{O}$, it can be always written as follows

$\dot{\mathbf{O}}=\mathbf{H} \dot{\mathbf{q}}$

where $\mathbf{H}$ is a $3 \times 3$ matrix whose explicit expression depends on the actual architecture of the $(3 \mathrm{~W}) \mathrm{S}$ limb.

With reference to Figs. 2 and 5, if the (3W)S limb is the RRPS limb of Figs. 2a and 5a the following relationship holds

$\dot{\mathbf{O}}=\dot{\mathrm{d}}_{0} \cdot \frac{\left(\mathbf{O}-\mathbf{A}_{0}\right)}{\mathrm{d}_{0}}+\left(\dot{\varphi}_{1} \mathbf{v}_{1}+\dot{\varphi}_{2} \mathbf{v}_{2}\right) \times\left(\mathbf{O}-\mathbf{A}_{0}\right)$ 
which yields $\dot{\mathbf{q}}=\left(\dot{\mathrm{d}}_{0}, \dot{\varphi}_{1}, \dot{\varphi}_{2}\right)^{\mathrm{T}}$ and

$$
\mathbf{H}=\left[\frac{\left(\mathbf{O}-\mathbf{A}_{0}\right)}{\mathrm{d}_{0}}, \mathbf{v}_{1} \times\left(\mathbf{O}-\mathbf{A}_{0}\right), \mathbf{v}_{2} \times\left(\mathbf{O}-\mathbf{A}_{0}\right)\right]
$$

If the (3W)S limb is the $\underline{\mathrm{R}} \perp(2 \underline{\mathrm{R}} \| \mathrm{R}) \mathrm{S}$ limb of Figs. $2 \mathrm{~b}$ and $5 \mathrm{~b}$, the following system of three scalar equations holds $^{1}$

$$
\dot{\mathbf{O}} \cdot \mathbf{v}_{2}=\dot{\varphi}_{1} \frac{\left|\left(\mathbf{O}-\mathbf{A}_{0}\right) \times\left(\mathbf{A}_{3}-\mathbf{A}_{0}\right)\right|}{\mathrm{e}}, \quad \dot{\mathbf{O}} \cdot \mathbf{r}_{0}=\dot{\varphi}_{2} \mathrm{k}_{0}, \quad \dot{\mathbf{O}} \cdot \mathbf{r}_{3}=\dot{\varphi}_{3} \mathrm{k}_{3}
$$

where $\mathbf{r}_{\mathrm{i}}=\frac{\left(\mathbf{O}-\mathbf{C}_{\mathrm{i}}\right)}{\mathrm{d}_{\mathrm{i}}}, \mathrm{k}_{\mathrm{i}}=\left[\mathbf{v}_{2} \times\left(\mathbf{C}_{\mathrm{i}}-\mathbf{A}_{\mathrm{i}}\right)\right] \cdot \mathbf{r}_{\mathrm{i}}$ for $\mathrm{i}=0,3$ and $\mathbf{v}_{2}=\frac{\mathbf{r}_{3} \times \mathbf{r}_{0}}{\left|\mathbf{r}_{3} \times \mathbf{r}_{0}\right|}$, whose explicit-form solution is

$$
\dot{\mathbf{O}}=\frac{1}{\mathbf{v}_{2} \cdot\left(\mathbf{r}_{3} \times \mathbf{r}_{0}\right)}\left[\dot{\varphi}_{1} \frac{\left|\left(\mathbf{O}-\mathbf{A}_{0}\right) \times\left(\mathbf{A}_{3}-\mathbf{A}_{0}\right)\right|}{\mathrm{e}}\left(\mathbf{r}_{3} \times \mathbf{r}_{0}\right)-\dot{\varphi}_{2} \mathrm{k}_{0}\left(\mathbf{r}_{3} \times \mathbf{v}_{2}\right)+\dot{\varphi}_{3} \mathrm{k}_{3}\left(\mathbf{r}_{0} \times \mathbf{v}_{2}\right)\right]
$$

Equation (12) yields, for the $\underline{\mathrm{R}} \perp(2 \underline{\mathrm{R}} \| \mathrm{R}) \mathrm{S} \operatorname{limb}, \dot{\mathbf{q}}=\left(\dot{\varphi}_{1}, \dot{\varphi}_{2}, \dot{\varphi}_{3}\right)^{\mathrm{T}}$ and

$$
\mathbf{H}=\frac{1}{\mathbf{v}_{2} \cdot\left(\mathbf{r}_{3} \times \mathbf{r}_{0}\right)}\left[\frac{\left|\left(\mathbf{O}-\mathbf{A}_{0}\right) \times\left(\mathbf{A}_{3}-\mathbf{A}_{0}\right)\right|}{\mathrm{e}}\left(\mathbf{r}_{3} \times \mathbf{r}_{0}\right),-\mathrm{k}_{0}\left(\mathbf{r}_{3} \times \mathbf{v}_{2}\right), \mathrm{k}_{3}\left(\mathbf{r}_{0} \times \mathbf{v}_{2}\right)\right]
$$

If the (3W)S limb is the $\underline{R} \perp(2 R \underline{P}) S$ limb of Figs. $2 \mathrm{c}$ and $5 \mathrm{c}$, the following system of three scalar equations holds

$$
\dot{\mathbf{O}} \cdot \frac{\left(\mathbf{O}-\mathbf{A}_{0}\right)}{\mathrm{d}_{0}}=\dot{\mathrm{d}}_{0}, \quad \dot{\mathbf{O}} \cdot \frac{\left(\mathbf{O}-\mathbf{A}_{3}\right)}{\mathrm{d}_{3}}=\dot{\mathrm{d}}_{3}, \quad \dot{\mathbf{O}} \cdot \mathbf{v}_{2}=\dot{\varphi}_{1} \frac{\left|\left(\mathbf{O}-\mathbf{A}_{0}\right) \times\left(\mathbf{A}_{3}-\mathbf{A}_{0}\right)\right|}{\mathrm{e}}
$$

whose explicit-form solution is

\footnotetext{
${ }^{1}$ It is worth noting (see Fig. 5b) that $\left(\mathbf{O}-\mathbf{C}_{\mathrm{i}}\right)=\left(\mathbf{O}-\mathbf{A}_{\mathrm{i}}\right)-\left(\mathbf{C}_{\mathrm{i}}-\mathbf{A}_{\mathrm{i}}\right)$, for $\mathrm{i}=0,3$, where $\left(\mathbf{O}-\mathbf{A}_{\mathrm{i}}\right)$ is known as a function of the platform pose and $\left(\mathbf{C}_{i}-\mathbf{A}_{i}\right)=g_{i}\left[\cos \varphi_{j} \mathbf{v}_{1}+\sin \varphi_{j}\left(\mathbf{v}_{2} \times \mathbf{v}_{1}\right)\right]$ with $j=2$ for $i=0$ and $j=i$ for $i=3$.
} 
$\dot{\mathbf{O}}=\frac{1}{\mathbf{v}_{2} \cdot\left(\mathbf{s}_{3} \times \mathbf{s}_{0}\right)}\left[-\dot{\mathrm{d}}_{0}\left(\mathbf{s}_{3} \times \mathbf{v}_{2}\right)+\dot{\mathrm{d}}_{3}\left(\mathbf{s}_{0} \times \mathbf{v}_{2}\right)+\dot{\varphi}_{1} \frac{\left|\left(\mathbf{O}-\mathbf{A}_{0}\right) \times\left(\mathbf{A}_{3}-\mathbf{A}_{0}\right)\right|}{\mathrm{e}}\left(\mathbf{s}_{3} \times \mathbf{s}_{0}\right)\right]$

where $\mathbf{s}_{\mathrm{i}}=\frac{\left(\mathbf{O}-\mathbf{A}_{\mathrm{i}}\right)}{\mathrm{d}_{\mathrm{i}}}$ for $\mathrm{i}=0,3$ and $\mathbf{v}_{2}=\frac{\mathbf{s}_{3} \times \mathbf{s}_{0}}{\left|\mathbf{s}_{3} \times \mathbf{s}_{0}\right|}$. Equation (15) yields, for the $\underline{\mathrm{R}} \perp(2 \mathrm{R} \underline{\mathrm{P}}) \mathrm{S} \operatorname{limb}, \dot{\mathbf{q}}=\left(\dot{\mathrm{d}}_{0}, \dot{\mathrm{d}}_{3}, \dot{\varphi}_{1}\right)^{\mathrm{T}}$ and

$$
\mathbf{H}=\frac{1}{\mathbf{v}_{2} \cdot\left(\mathbf{s}_{3} \times \mathbf{s}_{0}\right)}\left[-\left(\mathbf{s}_{3} \times \mathbf{v}_{2}\right),\left(\mathbf{s}_{0} \times \mathbf{v}_{2}\right), \frac{\left|\left(\mathbf{O}-\mathbf{A}_{0}\right) \times\left(\mathbf{A}_{3}-\mathbf{A}_{0}\right)\right|}{\mathrm{e}}\left(\mathbf{s}_{3} \times \mathbf{s}_{0}\right)\right]
$$

Regarding the platform angular velocity, $\omega$, the constraints it has to satisfy can be deduced as follows. When all the actuators are locked, the RS limb (see Figs. 1 and 4) yields the following constraints on the velocity of point $\mathrm{B}_{1}, \dot{\mathbf{B}}_{1}\left[\equiv \dot{\mathbf{O}}+\boldsymbol{\omega} \times\left(\mathbf{B}_{1}-\mathbf{O}\right)\right.$, when considered a platform point $]$

$$
\begin{aligned}
& 0=\dot{\mathbf{B}}_{1} \cdot \mathbf{u}_{2} \equiv\left[\dot{\mathbf{O}}+\boldsymbol{\omega} \times\left(\mathbf{B}_{1}-\mathbf{O}\right)\right] \cdot \mathbf{u}_{2} \equiv \mathbf{u}_{2} \cdot \dot{\mathbf{O}}+\left[\left(\mathbf{B}_{1}-\mathbf{O}\right) \times \mathbf{u}_{2}\right] \cdot \boldsymbol{\omega} \\
& 0=\dot{\mathbf{B}}_{1} \cdot\left(\mathbf{B}_{1}-\mathbf{A}_{1}\right) \equiv\left[\dot{\mathbf{O}}+\boldsymbol{\omega} \times\left(\mathbf{B}_{1}-\mathbf{O}\right)\right] \cdot\left(\mathbf{B}_{1}-\mathbf{A}_{1}\right) \equiv\left(\mathbf{B}_{1}-\mathbf{A}_{1}\right) \cdot \dot{\mathbf{O}}+\left[\left(\mathbf{B}_{1}-\mathbf{O}\right) \times\left(\mathbf{B}_{1}-\mathbf{A}_{1}\right)\right] \cdot \boldsymbol{\omega}
\end{aligned}
$$

whereas the US limb (see Figs. 1 and 4) yields the following constraint on the velocity of point $\mathrm{B}_{2}, \dot{\mathbf{B}}_{2}$ $\left[\equiv \dot{\mathbf{O}}+\boldsymbol{\omega} \times\left(\mathbf{B}_{2}-\mathbf{O}\right)\right.$, when considered a platform point $]$

$0=\dot{\mathbf{B}}_{2} \cdot\left(\mathbf{B}_{2}-\mathbf{A}_{2}\right) \equiv\left[\dot{\mathbf{O}}+\boldsymbol{\omega} \times\left(\mathbf{B}_{2}-\mathbf{O}\right)\right] \cdot\left(\mathbf{B}_{2}-\mathbf{A}_{2}\right) \equiv\left(\mathbf{B}_{2}-\mathbf{A}_{2}\right) \cdot \dot{\mathbf{O}}+\left[\left(\mathbf{B}_{2}-\mathbf{O}\right) \times\left(\mathbf{B}_{2}-\mathbf{A}_{2}\right)\right] \cdot \boldsymbol{\omega}$

As a consequence, when the actuators are not locked any longer, the zeros at the left-hand sides of Eqs. (17) can be replaced only by linear and homogeneous expressions in $\dot{\xi}=(\dot{x}, \dot{y})^{\mathrm{T}}$ and $\dot{z}$ whose coefficients depend on the type of kinematic chain which generates the RS or the US limbs; whereas, $\dot{\mathbf{O}}$ can be replaced only by expression (8). The result is that, in general, the following system of three scalar equations holds

$$
\begin{aligned}
& {\left[\left(\mathbf{B}_{1}-\mathbf{O}\right) \times \mathbf{u}_{2}\right] \cdot \boldsymbol{\omega}=\mathrm{m}_{11} \dot{\mathrm{x}}+\mathrm{m}_{12} \dot{\mathrm{y}}-\mathbf{u}_{2}{ }^{\mathrm{T}} \mathbf{H} \dot{\mathbf{q}}} \\
& {\left[\left(\mathbf{B}_{1}-\mathbf{O}\right) \times\left(\mathbf{B}_{1}-\mathbf{A}_{1}\right)\right] \cdot \boldsymbol{\omega}=\mathrm{m}_{21} \dot{\mathrm{x}}+\mathrm{m}_{22} \dot{\mathrm{y}}-\left(\mathbf{B}_{1}-\mathbf{A}_{1}\right)^{\mathrm{T}} \mathbf{H} \dot{\mathbf{q}}}
\end{aligned}
$$


System (18) can be put in matrix form as follows:

$\mathbf{N} \boldsymbol{\omega}=-\mathbf{D H} \dot{\mathbf{q}}+\mathbf{M} \dot{\mathbf{p}}$

where $\dot{\mathbf{p}}=(\dot{\mathrm{x}}, \dot{\mathrm{y}}, \dot{\mathrm{z}})^{\mathrm{T}}, \quad \mathbf{N}=\left[\left(\mathbf{B}_{1}-\mathbf{O}\right) \times \mathbf{u}_{2},\left(\mathbf{B}_{1}-\mathbf{O}\right) \times\left(\mathbf{B}_{1}-\mathbf{A}_{1}\right),\left(\mathbf{B}_{2}-\mathbf{O}\right) \times\left(\mathbf{B}_{2}-\mathbf{A}_{2}\right)\right]^{\mathrm{T}}, \mathbf{D}=\left[\mathbf{u}_{2},\left(\mathbf{B}_{1}-\mathbf{A}_{1}\right),\left(\mathbf{B}_{2}-\mathbf{A}_{2}\right)\right]^{\mathrm{T}}$ and $\mathbf{M}=\left[\mathbf{m}_{1}, \mathbf{m}_{2}, \mathbf{m}_{3}\right]$ with $\mathbf{m}_{1}=\left(\mathrm{m}_{11}, \mathrm{~m}_{21}, 0\right)^{\mathrm{T}}, \mathbf{m}_{2}=\left(\mathrm{m}_{12}, \mathrm{~m}_{22}, 0\right)^{\mathrm{T}}$ and $\mathbf{m}_{3}=(0,0, \mathrm{n})^{\mathrm{T}}$.

Appendix B reports the general expressions of the entries of matrix $\mathbf{M}$ for the ten above-identified types of kinematic chains that generate either an RS or a US limb when the actuators are locked. With reference to the formulas reported in Appendix B, the entries of this matrix for the RRPS-RRPS-UPS architecture of Fig. 3 ( $\mathbf{u}_{1}$ is chosen parallel to the axis of the actuated R pair of the $\underline{R} R \underline{P} S \operatorname{limb}, \mathrm{x}=\theta_{1}, \mathrm{y}=\mathrm{d}_{1}$ and $\mathrm{z}=\mathrm{d}_{2}$ ) are

$$
\begin{aligned}
& \mathrm{m}_{11}=\mathrm{d}_{1} \sin \theta_{2} ; \quad \mathrm{m}_{12}=0 \\
& \mathrm{~m}_{21}=0 ; \quad \mathrm{m}_{22}=\mathrm{d}_{1} ; \quad \mathrm{n}=\mathrm{d}_{2}
\end{aligned}
$$

\subsection{Singularity Analysis}

Equations (8) and (19) constitute the instantaneous input-output relationship that, out of singularities, states a one-to-one correspondence between the platform twist, $\left(\dot{\mathbf{O}}^{\mathrm{T}}, \boldsymbol{\omega}^{\mathrm{T}}\right)^{\mathrm{T}}$, and the six-tuple, $\left(\dot{\mathbf{q}}^{\mathrm{T}}, \dot{\mathbf{p}}^{\mathrm{T}}\right)^{\mathrm{T}}$, of the actuatedjoint rates. This relationship can be used to identify DPM's singularities, that is, the DPM configurations where the correspondence between $\left(\dot{\mathbf{O}}^{\mathrm{T}}, \boldsymbol{\omega}^{\mathrm{T}}\right)^{\mathrm{T}}$ and $\left(\dot{\mathbf{q}}^{\mathrm{T}}, \dot{\mathbf{p}}^{\mathrm{T}}\right)^{\mathrm{T}}$ is not one-to-one any longer.

Three types of PMs' singularities can be identified relying on the instantaneous input-output relationship [32, 33]: (I) PM configurations where the actuated-joint rates are indeterminate even though the platform twist is assigned, (II) PM configurations where the platform twist is indeterminate even though the actuated-joint rates are assigned, and (III) PM configurations where both the two previous singularity conditions are satisfied. This singularity classification can be further refined by taking into account the rates of the non-actuated joints [34].

Type-I singularities occur at the workspace boundaries; whereas, type-II singularities are mainly located inside the workspace and identify configurations where at least one link has to stand an infinite internal load to equilibrate any (even very small) external load applied to the platform. As a consequence, type-II singularities, must be always identified during design and carefully avoided during functioning. 
PMs' input-output relationships are always linear and homogeneous systems, in the platform twist and the actuated-joint rates, whose coefficient matrices depend only on the actuated-joint variables [32, 33]. Therefore, in six-dof PMs, type-I singularities occur when the determinant of the $6 \times 6$ coefficient matrix that multiplies the actuated-joint rates is equal to zero, type-II singularities occur when the determinant of the $6 \times 6$ coefficient matrix that multiplies the platform twist is equal to zero and type-III singularities occur when these two determinants are simultaneously equal to zero.

With reference to the above-deduced input-output relationship, these results bring to conclude that, in the studied DPMs, a type-I singularity occurs when

$\operatorname{det}(\mathbf{H}) \operatorname{det}(\mathbf{M})=0$

whereas a type-II singularity occurs when

$\operatorname{det}(\mathbf{G}) \operatorname{det}(\mathbf{N})=0$

where the expression of $\mathbf{G}$ depends on the architecture of the $(3 \mathrm{~W}) \mathrm{S}$ limb: it is equal to the $3 \times 3$ identity matrix for the RRPS limb of Fig. $2 \mathrm{a}, \mathbf{G}=\left[\mathbf{v}_{2}, \mathbf{r}_{3}, \mathbf{r}_{0}\right]$ for the single-loop $\underline{\mathrm{R}} \perp(2 \underline{\mathrm{R}} \| \mathrm{R}) \mathrm{S}$ limb of Fig. $2 \mathrm{~b}$, and $\mathbf{G}=\left[\mathbf{v}_{2}, \mathbf{s}_{3}, \mathbf{s}_{0}\right]$ for the single-loop $\underline{\mathrm{R}} \perp(2 \mathrm{R} \underline{\mathrm{P}}) \mathrm{S}$ limb of Fig. 2c.

Equation (21) is satisfied if and only if either

$\operatorname{det}(\mathbf{H})=0$;

or

$\operatorname{det}(\mathbf{M}) \equiv \mathrm{n}\left(\mathrm{m}_{11} \mathrm{~m}_{22}-\mathrm{m}_{21} \mathrm{~m}_{12}\right)=0$

Equation (23) identifies DPM configurations (shoulder singularities) where the velocity $\dot{\mathbf{O}}$ cannot assume any value since the image of the linear mapping defined by Eq. (8) is not three-dimensional (i.e., the workspace boundary of the (3W)S limb has been reached). Equation (24) identifies DPM configurations (wrist singularities) where the platform angular velocity $\omega$ cannot assume any value when $\dot{\mathbf{O}}=0$ since the image of the 
linear mapping defined by Eq. (19) with $\dot{\mathbf{q}}=\mathbf{0}$ is not three-dimensional (i.e., the workspace boundaries of the kinematic chains which generate either the RS or the US limbs have been reached).

For the RRPS-RRPS-UPS architecture of Fig. 3, Eqs. (9) and (10) reveal that Eq. (23) is satisfied if $\mathrm{d}_{0}$ is equal to zero (i.e., if $\mathrm{A}_{0}$ coincides with $\mathrm{O}$ (see Fig. 5a)); whereas, Eqs. (20a) and (20b) yield

$\operatorname{det}(\mathbf{M}) \equiv \mathrm{d}_{2} \mathrm{~d}_{1}^{2}$

and Eq. (24) is satisfied if either $\mathrm{d}_{1}$ or $\mathrm{d}_{2}$ are equal to zero.

Regarding type-II singularities, Eq. (22) is satisfied if and only if either

$\operatorname{det}(\mathbf{G})=0$

or

$\operatorname{det}(\mathbf{N})=0$

Equation (26) identifies DPM configurations (translation singularities) where $\dot{\mathbf{O}}$ can be different from zero even though the actuated joints of the (3W)S limb are locked (i.e., even though $\dot{\mathbf{q}}=\mathbf{0}$ (see Eq. (8))). Therefore, they are limb singularities of the (3W)S limb. In particular, the RRPS limb (Figs. 2a and 5a) yields no type-II singularity since its matrix $\mathbf{G}$ is the $3 \times 3$ identity matrix. Also, the single-loop $\underline{\mathrm{R}} \perp(2 \underline{\mathrm{R}} \| \mathrm{R}) \mathrm{S}$ limb (Figs. $2 \mathrm{~b}$ and 5b), whose matrix $\mathbf{G}$ is $\left[\mathbf{v}_{2}, \mathbf{r}_{3}, \mathbf{r}_{0}\right]$ with $\operatorname{det}(\mathbf{G})=\mathbf{v}_{2} \cdot\left(\mathbf{r}_{3} \times \mathbf{r}_{0}\right)$, yields type-II singularities if and only if the two unit vectors $\mathbf{r}_{3}$ and $\mathbf{r}_{0}$ are parallel, that is, when the points $\mathrm{C}_{0}, \mathrm{C}_{3}$ and $\mathrm{O}$ of Fig. $5 \mathrm{~b}$ are aligned (Fig. 6a); whereas, the single-loop $\underline{\mathrm{R}} \perp(2 \mathrm{R} \underline{\mathrm{P}}) \mathrm{S} \operatorname{limb}$ (Figs. $2 \mathrm{c}$ and $5 \mathrm{c}$ ) whose matrix $\mathbf{G}$ is $\left[\mathbf{v}_{2}, \mathbf{s}_{3}, \mathbf{s}_{0}\right]$ with $\operatorname{det}(\mathbf{G})=\mathbf{v}_{2} \cdot\left(\mathbf{s}_{3} \times \mathbf{s}_{0}\right)$, yields typeII singularities if and only if the two unit vectors $\mathbf{s}_{3}$ and $\mathbf{s}_{0}$ are parallel, that is, when the points $\mathrm{A}_{0}, \mathrm{~A}_{3}$ and $\mathrm{O}$ of Fig. 5c are aligned (Fig. 6b).

Equation (27) identifies DPM configurations (rotation singularities) where the platform angular velocity $\omega$ can be different from zero even though all the actuated joints are locked (i.e., even though $\dot{\mathbf{q}}=\mathbf{0}$ and $\dot{\mathbf{p}}=\mathbf{0}$ (see Eq. (19)). Therefore, they are configurations where the geometry of the generated S-RS-US structure is singular. The above-deduced analytic expression of matrix $\mathbf{N}$ yields 
Expression (28) is equal to zero (i.e., Eq. (27) is satisfied), if either

(a) the vector in curly brackets is a null vector, or

(b) the R-pair axis (i.e., the line through $\mathrm{A}_{1}$ and parallel to $\mathbf{u}_{2}$ in Fig. 4) is parallel to the line passing through $\mathrm{O}$ and $\mathrm{B}_{1}$ (in this case $\left(\mathbf{B}_{1}-\mathbf{O}\right) \times \mathbf{u}_{2}$ is a null vector), or

(c) the direction of the vector in curly brackets (i.e., of the intersection line between the planes of the two triangles $A_{1} B_{1} O$ and $A_{2} B_{2} O$ (Fig. 4)), the R-pair axis (i.e., the line through $A_{1}$ and parallel to $\mathbf{u}_{2}$ in Fig. 4) and the line passing through $\mathrm{O}$ and $\mathrm{B}_{1}$ are parallel to a unique plane.

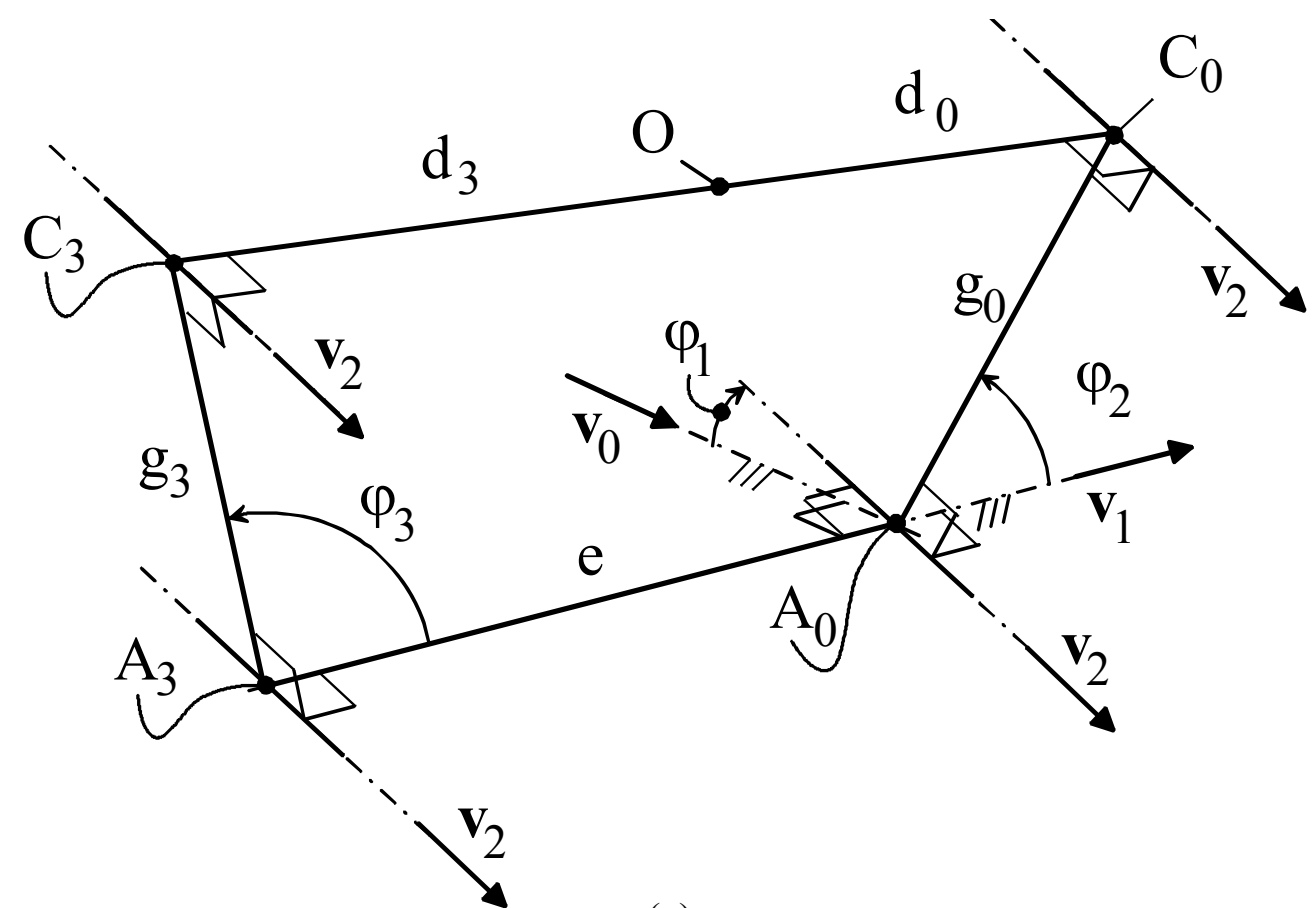

(a)

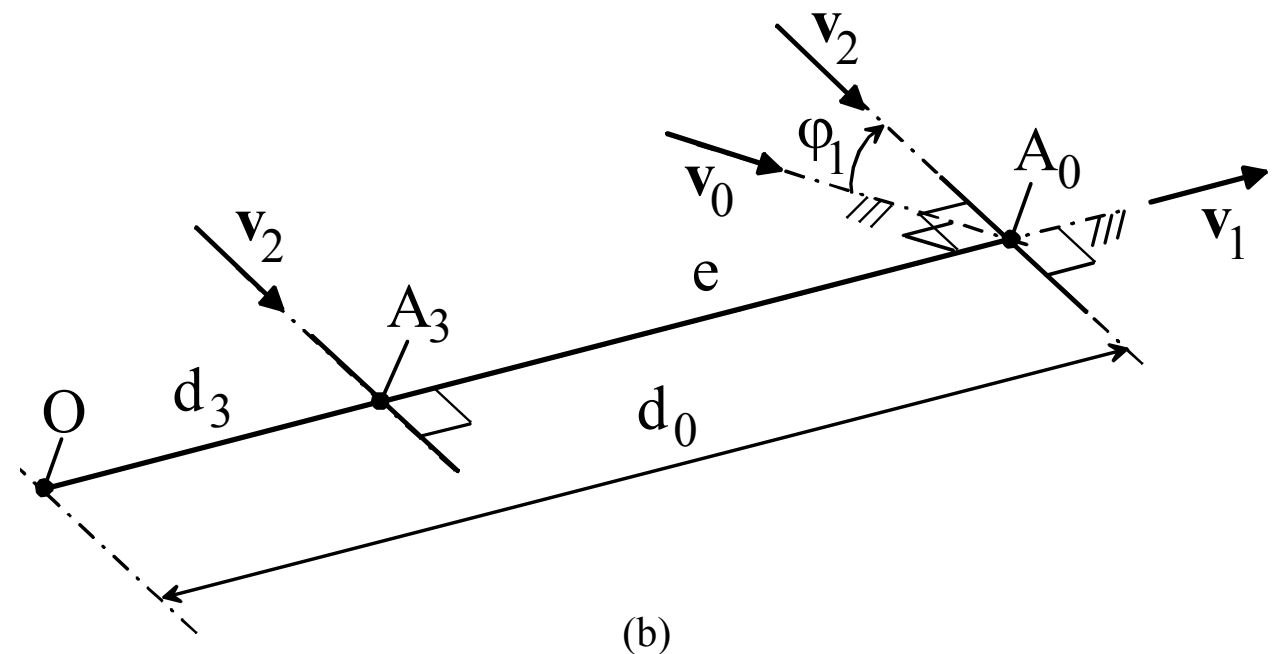

Figure 6: Translation singularities: geometric conditions identifying a limb singularity (a) of the single-loop $\underline{\mathrm{R}} \perp(2 \underline{\mathrm{R}}|| \mathrm{R}) \mathrm{S}$ limb and (b) of the single-loop $\underline{\mathrm{R}} \perp(2 \mathrm{R} \underline{\mathrm{P}}) \mathrm{S}$ limb. 
With reference to Fig. 4 , condition (a) occurs when either $d_{1}$ or $d_{2}$ are equal to zero or the two triangles $A_{1} B_{1} O$ and $\mathrm{A}_{2} \mathrm{~B}_{2} \mathrm{O}$ are coplanar; whereas, condition (c) occurs when either the R-pair axis lies on the plane of the triangle $\mathrm{A}_{1} \mathrm{~B}_{1} \mathrm{O}$ or the triangle $\mathrm{B}_{1} \mathrm{OB}_{2}$, fixed to the platform, and the triangle $\mathrm{A}_{2} \mathrm{~B}_{2} \mathrm{O}$ are flattened on a single plane (Fig. 7). The platform can perform an infinitesimal rotation around the intersection line between the planes of the two triangles $\mathrm{A}_{1} \mathrm{~B}_{1} \mathrm{O}$ and $\mathrm{A}_{2} \mathrm{~B}_{2} \mathrm{O}$ when conditions (b) or (c) occur.

Eventually, it is worth noting that, since all the singularity conditions which require the zeroing of a limb length (i.e., of $\mathrm{d}_{0}$ or $\mathrm{d}_{1}$ or $\mathrm{d}_{2}$ ) cannot occur in practice, the type-II singularities of the $\underline{\mathrm{RRPS}}-\underline{\mathrm{R} R} \underline{\mathrm{P} S} \mathrm{~S}-\mathrm{UPS}$ architecture of Fig. 3 are in practice only the configurations where either the two triangles $A_{1} B_{1} O$ and $A_{2} B_{2} O$ are coplanar or conditions (b) and (c) are satisfied.

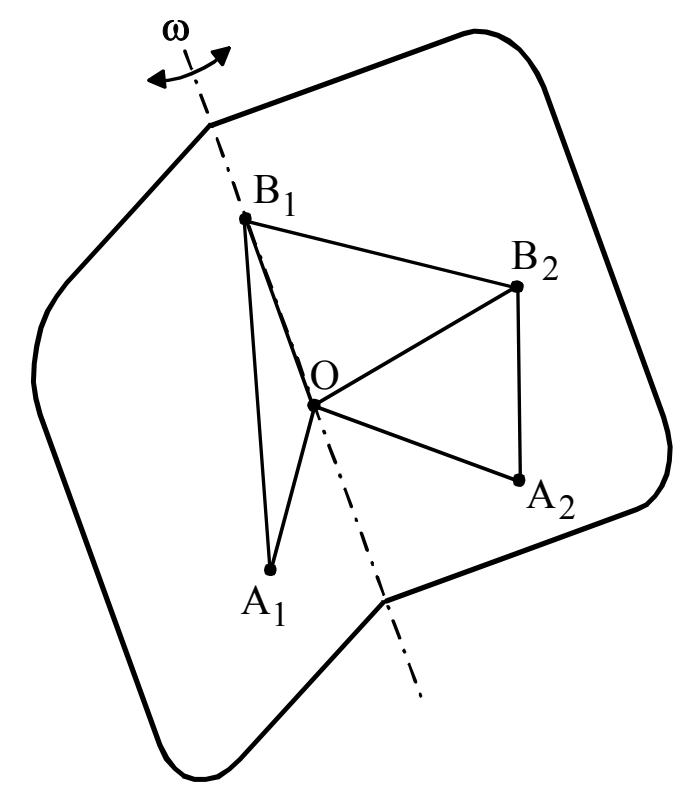

Figure 7: Rotation singularities: a configuration that satisfies geometric condition (c).

\section{Discussion}

Even though the performance analysis of an actual model of one out of the 63 identified architectures is out of the scope of this paper, the above-deduced general relationships will be used here to give some hints on how could be workspace, statics behavior, positioning precision and isotropy/stiffness of these architectures.

Regarding their dynamic performances, since an actual model is necessary to evaluate the maximum accelerations of the platform, they will not be considered here. Nevertheless, it is worth reminding that all the 63 architectures allow the actuators to be put on (or near) the base and that, since this is the key requirement to 
drastically reduce the mobile masses and to get high end-effector's accelerations (see, for instance, [18]), they are all expected to be able to reach high platform's accelerations.

\subsection{Workspace:}

In general, the simple reduction of the limb number is not sufficient to guarantee an increased workspace when different architectures/limbs are considered. Nevertheless, a reduced limb number always [25] yields a reduced number ${ }^{2}$ of analytic conditions (inequalities) that bound the volume where, at constant platform orientation, the platform reference point must be located. Also, such reduction of the inequality system that bound the constantorientation workspace is the premise to get an increased volume, if it is suitably exploited during design.

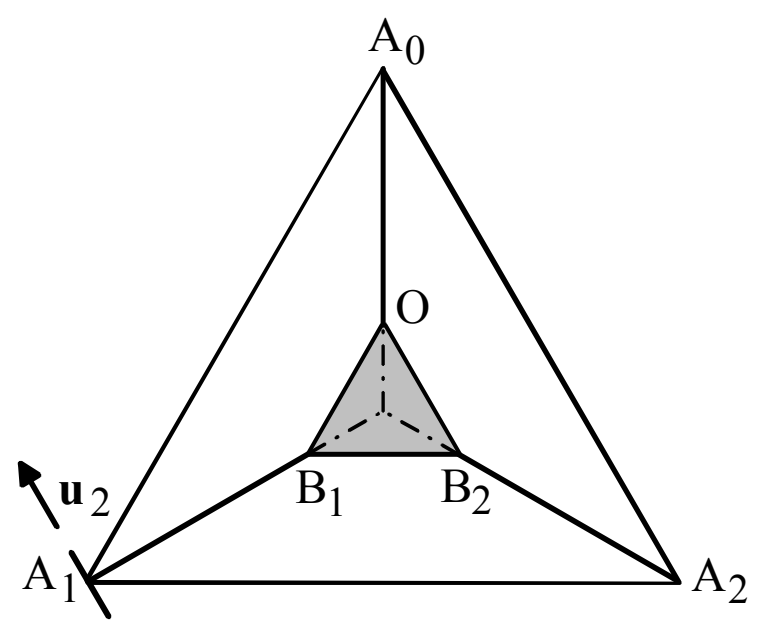

Figure 8: Studied RRPS-RRPS-UPS architecture: top view of the home position.

Just to give an idea of how wide could be the constant orientation workspace of the proposed architectures, the

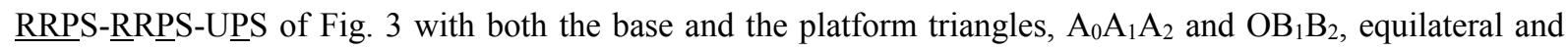
with the ratio between the sides of $\mathrm{OB}_{1} \mathrm{~B}_{2}$ and $\mathrm{A}_{0} \mathrm{~A}_{1} \mathrm{~A}_{2}$ equal to 0.25 is analyzed. Hereafter, the length unit (1.u.) is assumed equal to the side length of the base triangle $\mathrm{A}_{0} \mathrm{~A}_{1} \mathrm{~A}_{2}$. Also, its home position is defined as the configuration where the three limb axes are the lateral edges of a trirectangular tetrahedron, with $\mathrm{A}_{0} \mathrm{~A}_{1} \mathrm{~A}_{2}$ as base, where the triangles $A_{0} A_{1} A_{2}$ and $O B B_{1} B_{2}$ are parallel to each other (see Fig. 8) and the limb lengths, $d_{0}, d_{1}$ and $\mathrm{d}_{2}$ (see Figs. 4 and 5(a)), are all equal to 0.53 1.u.

It is easy to check through either formula (28) or a simple geometric reasoning based on the vectors appearing in formula (28) that the home position is not a singular configuration. Also, the same geometric reasoning makes it

\footnotetext{
2 Gosselin [25] demonstrated that, if the constant orientation workspace of fully-parallel manipulators is considered, each UPS limb yields two concentric spheres, with radii equal to the minimum and the maximum limb length, which delimit the volume where the platform reference point must be located.
} 


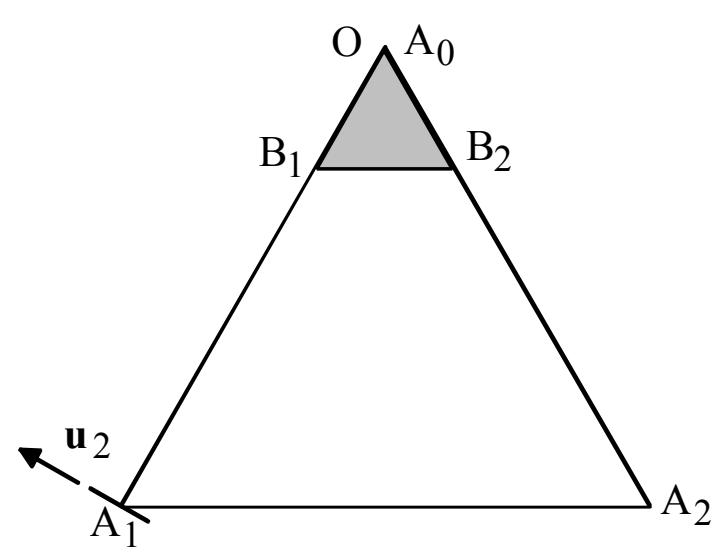

(a)

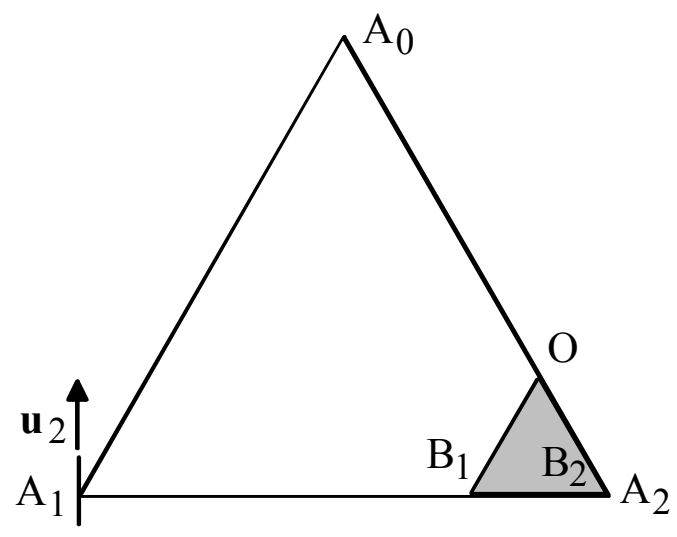

(c)

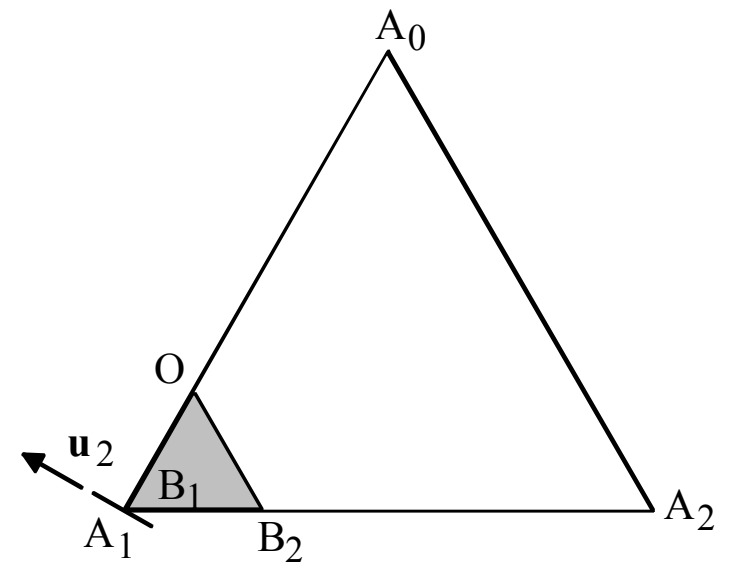

(b)

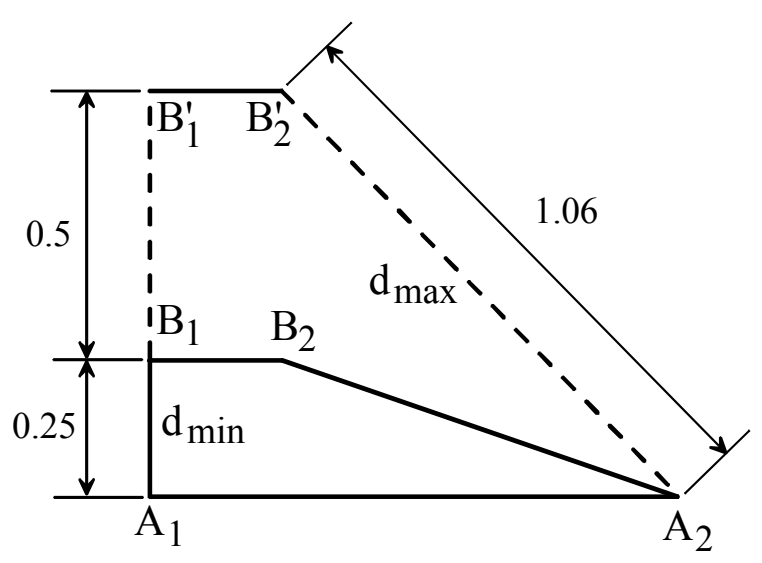

(d)

Figure 9: Singularity-free workspace of the studied RRPS-RRPS-UPS: top views of the constant-platformorientation configurations where (a) points $A_{0}$ and $O$, (b) points $A_{1}$ and $B_{1}$, and (c) points $A_{2}$ and $B_{2}$ lie on the same vertical line; (d) front view of the configuration (b) with the minimum and maximum limb lengths necessary to make the platform translate of 0.5 l.u. along its normal.

possible to demonstrate that the three constant-platform-orientation configurations shown in Figs. 9(a), 9(b) and 9(c) are not singular provided that the platform height on the base is different from zero and that the same holds for all the constant-platform-orientation configurations where, in the top view, the platform triangle $\mathrm{OB}_{1} \mathrm{~B}_{2}$ is inside the base triangle $\mathrm{A}_{0} \mathrm{~A}_{1} \mathrm{~A}_{2}$. Eventually, Fig. $9(\mathrm{~d})$ shows that the platform height can range from $0.251 . u$. to 0.75 l.u., if the limb lengths $\mathrm{d}_{0}, \mathrm{~d}_{1}$ and $\mathrm{d}_{2}$ can vary from 0.25 1.u. to 1.06 l.u. The conclusion is that, by assuming reasonable variations of the limb lengths, a triangular prism with height equal to $0.51 . u$. and cross section equal to the base triangle $\mathrm{A}_{0} \mathrm{~A}_{1} \mathrm{~A}_{2}$ can be identified which is free from singularities. This wide constant-orientation workspace may be reduced when, during design, specific conditions on the platform orientation variation and/or on the distance from singularities are given. Such a detailed analysis is out of the scope of this paper.

\subsection{Statics and Positioning Precision:}

Equations (8) and (19) can be put in the matrix form $\left(\mathbf{0}_{3}\right.$ is the $3 \times 3$ null matrix $)$ 


$$
\left\{\begin{array}{c}
\dot{\mathbf{q}} \\
\dot{\mathbf{p}}
\end{array}\right\}=\mathbf{J}\left\{\begin{array}{c}
\dot{\mathbf{O}} \\
\boldsymbol{\omega}
\end{array}\right\}, \quad \text { where } \quad \mathbf{J}=\left[\begin{array}{cc}
\mathbf{H}^{-1} & \mathbf{0}_{3} \\
\mathbf{M}^{-1} \mathbf{D} & \mathbf{M}^{-1} \mathbf{N}
\end{array}\right] \quad \text { with } \quad \mathbf{J}^{-1}=\left[\begin{array}{cc}
\mathbf{H} & \mathbf{0}_{3} \\
-\mathbf{N}^{-1} \mathbf{D H} & \mathbf{N}^{-1} \mathbf{M}
\end{array}\right],
$$

Equation (29) and the virtual work principle [35,36] yield the following input-output static relationship:

$$
\$_{\mathrm{O}}=\mathbf{J}^{\mathrm{T}} \tau
$$

where $\$_{\mathrm{O}}\left[\equiv\left(\mathbf{f}^{\mathrm{T}}, \mathbf{n}^{\mathrm{T}}\right)^{\mathrm{T}}\right]$ is the wrench collecting the resultant force, $\mathbf{f}$, and the resultant moment, $\mathbf{n}$, about $\mathrm{O}$ of the force system the platform applies when interacting; whereas, $\tau\left[\equiv\left(\tau_{1}, \tau_{2}, \tau_{3}, \tau_{x}, \tau_{y}, \tau_{z}\right)^{T}\right]$ is the six-tuple collecting the generalized torques $\tau_{\mathrm{w}}\left[\equiv\left(\tau_{1}, \tau_{2}, \tau_{3}\right)^{\mathrm{T}}\right], \tau_{\mathrm{x}}, \tau_{\mathrm{y}}$ and $\tau_{\mathrm{z}}$ applied by the actuators of the $\underline{3 \mathrm{~W}}$ mechanism and of the $\underline{\mathrm{X}}, \underline{\mathrm{Y}}$ and $\underline{\mathrm{Z}}$ joints, respectively.

Also, Eq. (8) can be exploited to relate the actuation errors, $\Delta \mathbf{q}\left[\equiv\left(\Delta \mathrm{q}_{1}, \Delta \mathrm{q}_{2}, \Delta \mathrm{q}_{3}\right)^{\mathrm{T}}\right]$, in the $(\underline{3 \mathrm{~W}}) \mathrm{S}$ limb to the resulting position error, $\Delta \mathbf{O}$, of the platform. Actually, if the errors $\Delta \mathbf{q}$ and $\Delta \mathbf{O}$ replace the differentials $\mathrm{d} \mathbf{q}$ and dO, respectively, in Eq. (8) and $\mathbf{h}_{\mathbf{i}}$, for $\mathrm{i}=1,2,3$, denotes the $\mathrm{i}$-th column vector of matrix $\mathbf{H}$, the following inequality can be deduced from Eq. (8)

$\|\Delta \mathbf{O}\|_{2} \leq|| \mathbf{h}_{1}\left\|_{2}\left|\Delta \mathrm{q}_{1}\right|+\right\| \mathbf{h}_{2}\left\|_{2}\left|\Delta \mathrm{q}_{2}\right|+\right\| \mathbf{h}_{3} \|_{2}\left|\Delta \mathrm{q}_{3}\right|$

where $\|(\cdot)\|_{2}$ denotes the Euclidean vector norm [37] of (.). For the RRPS limb (Figs. 2(a) and 5(a)) of the RRPS-RRPS-UPS architecture (Fig. 3), Eqs. (9) and (10) yield the following explicit expression of inequality (31):

$\|\Delta \mathbf{O}\|_{2} \leq\left|\Delta \mathrm{d}_{0}\right|+\mathrm{d}_{0}\left|\Delta \varphi_{1} \sin \varphi_{2}\right|+\mathrm{d}_{0}\left|\Delta \varphi_{2}\right|$

which highlights that platform's position error increases with $\mathrm{d}_{0}$.

Eventually, the following inequality can be deduced from Eq. (19) by replacing the differentials with the corresponding actuation errors:

$|\Delta \psi| \leq\left\|\mathbf{N}^{-1} \mathbf{D}\right\|_{F}\|\Delta \mathbf{O}\|_{2}+\left\|\mathbf{N}^{-1} \mathbf{m}_{1}\right\|_{2}|\Delta \mathbf{x}|+\left\|\mathbf{N}^{-1} \mathbf{m}_{2}\right\|_{2}|\Delta \mathrm{y}|+\left\|\mathbf{N}^{-1} \mathbf{m}_{3}\right\|_{2}|\Delta \mathrm{z}|$ 
where $\mathbf{M}=\left[\mathbf{m}_{1}, \mathbf{m}_{2}, \mathbf{m}_{3}\right],\|(\cdot)\|_{F}$ denotes the Frobenius matrix norm [37] of $(\cdot)$ and $\Delta \psi(\equiv$ platform's orientation error) is the rotation angle associated to the rotation matrix ${ }^{3}$ that makes the actual platform orientation coincide with its desired orientation. For the $\underline{R R P S}$-RRPS-UPS architecture (see Figs. 3 and 4), Eqs. (20a) and (20b) yield the following explicit expression of inequality (33):

$|\Delta \psi| \leq\left\|\mathbf{N}^{-1} \mathbf{D}\right\|_{F}\|\Delta \mathbf{O}\|_{2}+\left\|\mathbf{v}_{1}\right\|_{2}\left|\mathrm{~d}_{1} \Delta \theta_{1} \sin \theta_{2}\right|+\left\|\mathbf{v}_{2}\right\|_{2}\left|\mathrm{~d}_{1} \Delta \mathrm{d}_{1}\right|+\left\|\mathbf{v}_{3}\right\|_{2}\left|\mathrm{~d}_{2} \Delta \mathrm{d}_{2}\right|$

where $\mathbf{N}^{-1}=\left[\mathbf{v}_{1}, \mathbf{v}_{2}, \mathbf{v}_{3}\right]$ with [see Eq. (28) for the explicit expression of $\left.\operatorname{det}(\mathbf{N})\right]$

$$
\begin{aligned}
& \mathbf{v}_{1}=\frac{\left[\left(\mathbf{B}_{1}-\mathbf{O}\right) \times\left(\mathbf{B}_{1}-\mathbf{A}_{1}\right)\right] \times\left[\left(\mathbf{B}_{2}-\mathbf{O}\right) \times\left(\mathbf{B}_{2}-\mathbf{A}_{2}\right)\right]}{\operatorname{det}(\mathbf{N})} \\
& \mathbf{v}_{2}=\frac{\left[\left(\mathbf{B}_{2}-\mathbf{O}\right) \times\left(\mathbf{B}_{2}-\mathbf{A}_{2}\right)\right] \times\left[\left(\mathbf{B}_{1}-\mathbf{O}\right) \times \mathbf{u}_{2}\right]}{\operatorname{det}(\mathbf{N})} \\
& \mathbf{v}_{3}=\frac{\left[\left(\mathbf{B}_{1}-\mathbf{O}\right) \times \mathbf{u}_{2}\right] \times\left[\left(\mathbf{B}_{1}-\mathbf{O}\right) \times\left(\mathbf{B}_{1}-\mathbf{A}_{1}\right)\right]}{\operatorname{det}(\mathbf{N})}
\end{aligned}
$$

Formulas (33), (34) and (35) highlight that platform's orientation error is inversely proportional to $|\operatorname{det}(\mathbf{N})|$ (i.e., the nearer the type-II singularity, the bigger the orientation error $|\Delta \psi|$ ).

\subsection{Stiffness and Isotropy:}

Manipulators' stiffness is usually studied by assuming $[23,35,36]$ that only the actuated joints are flexible in the structure generated by locking the actuators. Here, this hypothesis yields the following relationship:

$$
\tau=\operatorname{diag}(\mathbf{k})\left\{\begin{array}{l}
\Delta \mathbf{q} \\
\Delta \mathbf{p}
\end{array}\right\}
$$

where $\mathbf{k}\left[\equiv\left(\mathrm{k}_{1}, \mathrm{k}_{2}, \mathrm{k}_{3}, \mathrm{k}_{\mathrm{x}}, \mathrm{k}_{\mathrm{y}}, \mathrm{k}_{\mathrm{z}}\right)^{\mathrm{T}}\right]$ is the six-tuple collecting actuated joints' stiffness $\mathbf{k}_{\mathrm{w}}\left[\equiv\left(\mathrm{k}_{1}, \mathrm{k}_{2}, \mathrm{k}_{3}\right)^{\mathrm{T}}\right], \mathrm{k}_{\mathrm{x}}, \mathrm{k}_{\mathrm{y}}$ and $\mathrm{k}_{\mathrm{z}}$ of the $\underline{3 \mathrm{~W}}$ mechanism and of the $\underline{X}, \underline{\mathrm{Y}}$ and $\underline{\mathrm{Z}}$ joints, respectively.

\footnotetext{
${ }^{3}$ A rotation matrix, $\mathbf{Q}$, can be explicitly expressed [31, 35] as a function of the unit vector, $\mu$, of the rotation axis and of the rotation angle $\psi$ through the formula $\mathbf{Q}=\cos \psi \mathbf{I}_{3}+\sin \psi \mu^{\mathrm{Sk}}+(1-\cos \psi) \boldsymbol{\mu} \boldsymbol{\mu}^{\mathrm{T}}$, where $\mathbf{I}_{3}$ is the $3 \times 3$ identity matrix and $\mu^{\mathrm{Sk}}$ is the skew-symmetric matrix associated to the unit vector $\mu$. As a consequence, the vector $\Delta \psi=\mu \Delta \psi$, whose magnitude is $|\Delta \psi|$, uniquely locates the actual platform orientation with respect to the desired platform orientation.
} 
The introduction of Eq. (36) into Eq. (30) and, in the resulting expression, the replacement of $\Delta \mathbf{q}$ and $\Delta \mathbf{p}$ with their expressions given by Eq. (29), where the finite differences replace the differentials, yield

$\mathbf{S}_{\mathrm{O}}=\mathbf{J}^{\mathrm{T}} \operatorname{diag}(\mathbf{k}) \mathbf{J}\left\{\begin{array}{l}\Delta \mathbf{O} \\ \Delta \boldsymbol{\psi}\end{array}\right\}$

Equation (37) provides the following expression of the stiffness matrix, K, of the presented DPMs

$\mathbf{K}=\mathbf{J}^{\mathrm{T}} \operatorname{diag}(\mathbf{k}) \mathbf{J}$

In these DPMs, the analytic expression of $\mathbf{K}$, Eq. (38) provides, cannot be further simplified [23, 35, 36] to the form $\mathrm{kJ} \mathbf{J}^{\mathrm{T}} \mathbf{J}$ since their actuators are not of the same type. Nevertheless, a normalized expression [23, 31, 38] of the matrix $\mathbf{J}^{\mathrm{T}} \mathbf{J}$ obtained by dividing all the manipulator lengths by a characteristic length (e.g., b $b_{1}$ or $b_{2}$ of Fig. 4) still is important during design to find isotropic configurations (i.e., configurations where the input-output both static and instantaneous kinematic relationships do not depend on directions). The matrix $\mathbf{J}^{\mathrm{T}} \mathbf{J}$ of the studied DPMs has the following expression

$\mathbf{J}^{\mathrm{T}} \mathbf{J}=\left[\begin{array}{cc}\mathbf{H}^{-\mathrm{T}} & \mathbf{D}^{\mathrm{T}} \mathbf{M}^{-\mathrm{T}} \\ \mathbf{0}_{3} & \mathbf{N}^{\mathrm{T}} \mathbf{M}^{-\mathrm{T}}\end{array}\right]\left[\begin{array}{cc}\mathbf{H}^{-1} & \mathbf{0}_{3} \\ \mathbf{M}^{-1} \mathbf{D} & \mathbf{M}^{-1} \mathbf{N}\end{array}\right]=\left[\begin{array}{cc}\mathbf{H}^{-\mathrm{T}} \mathbf{H}^{-1}+\mathbf{D}^{\mathrm{T}} \mathbf{M}^{-\mathrm{T}} \mathbf{M}^{-1} \mathbf{D} & \mathbf{D}^{\mathrm{T}} \mathbf{M}^{-\mathrm{T}} \mathbf{M}^{-1} \mathbf{N} \\ \mathbf{N}^{\mathrm{T}} \mathbf{M}^{-\mathrm{T}} \mathbf{M}^{-1} \mathbf{D} & \mathbf{N}^{\mathrm{T}} \mathbf{M}^{-\mathrm{T}} \mathbf{M}^{-1} \mathbf{N}\end{array}\right]$

Anyway, the introduction of the $\Delta \mathbf{q}$ and $\Delta \mathbf{p}$ expressions deduced from Eq. (36), that is,

$$
\left\{\begin{array}{l}
\Delta \mathbf{q} \\
\Delta \mathbf{p}
\end{array}\right\}=\operatorname{diag}(\mathbf{k})^{-1} \tau
$$

into inequalities (31) and (33) figures out how flexible these DPMs could be. Indeed, the resulting formulas clearly shows that, at parity of actuated joints' stiffness, their stiffness worsens at the configurations where the positioning precision worsens, too (i.e, the nearer the type-II singularity, the worse both the stiffness and the positioning precision). 


\section{Conclusions}

Manipulators with fully or partially decoupled kinematics allow the use of simplified path-planning algorithms which sequentially involve a limited number of actuators and make the machine behave in an easy-to-visualize manner that facilitates the operator.

A novel family of decoupled parallel manipulators has been identified which contains sixty-three DPM architectures. The DPMs of this family feature only three limbs with connectivity six and all the actuators on or near to the base. These features make all the members of this family as fast as fully-parallel manipulators with complex kinematics without the use of cumbersome six-limbed architectures.

The position analysis of all the proposed DPMs has been solved through a unified approach and the demonstration that both the direct and the inverse position analyses of these DPMs are easily solvable in closed form has been provided.

Also, their instantaneous kinematics has been addressed and all the singularity conditions of these DPMs have been analytically determined and geometrically interpreted.

Eventually, one DPM architecture of the proposed family has been found which exhibits three limbs with the same topology and a much simple kinematics.

The obtained results provide all the tools necessary to design these DPMs and demonstrate that their control can be implemented by using analytical relationship with a clear geometric meaning. This and the fact that the DPMs of this family feature only three limbs with actuators on or near to the base make them interesting design options.

\section{Acknowledgments}

This work has been developed at the Laboratory of Advanced Mechanics (MECH-LAV) of Ferrara Technopole, supported by UNIFE and MIUR funds and by Regione Emilia Romagna (District Councillorship for Productive Assets, Economic Development, Telematic Plan) POR-FESR 2007-2013, Attività I.1.1.

\section{Appendix A}

The following type of trigonometric equation

$\mathrm{h}_{2} \cos \mathrm{x}+\mathrm{h}_{1} \sin \mathrm{x}+\mathrm{h}_{0}=0$, 
where the coefficients $h_{i}$ for $i=0,1,2$ are real constants and $x$ is the unknown, can be transformed into a quadratic algebraic equation in $\mathrm{t}=\tan (\mathrm{x} / 2)$ by introducing the trigonometric identities

$\cos x=\frac{1-t^{2}}{1+t^{2}}, \quad \sin x=\frac{2 t}{1+t^{2}}$

and, then, rationalizing the resulting expression.

This algebraic manipulation transforms Eq. (A1) as follows

$\left(h_{0}-h_{2}\right) t^{2}+2 h_{1} t+\left(h_{0}+h_{2}\right)=0$

Equation (A3) yields the following two explicit-form solutions

$\mathrm{t}=\frac{-\mathrm{h}_{1} \pm \sqrt{\mathrm{h}_{1}^{2}+\mathrm{h}_{2}^{2}-\mathrm{h}_{0}^{2}}}{\mathrm{~h}_{0}-\mathrm{h}_{2}}$

\section{Appendix B}

This appendix deduces some general expressions of the entries of the matrix $\mathbf{M}$ that appears in Eq. (19). Such expressions hold for all the above-identified limbs of type $\underline{X} \underline{\mathrm{Y}} \mathrm{S}, \underline{\mathrm{XY}} \mathrm{RS}, \mathrm{RXY} S, \underline{\mathrm{Z} U S}$ and UZ $\underline{\mathrm{Z}}$. Hereafter, $\mathrm{x}, \mathrm{y}$ and $\mathrm{z}$ denote the actuated-joint variables of the actuated pairs $\underline{X}, \underline{Y}$ and $\underline{Z}$, respectively.

With reference to the RS limb of Fig. 4, the first formula of Eqs. (1b) yields

$\mathbf{B}_{1}=\mathbf{A}_{1}+\mathrm{d}_{1}\left[\mathbf{u}_{1} \cos \theta_{2}+\left(\mathbf{u}_{2} \times \mathbf{u}_{1}\right) \sin \theta_{2}\right]$

where $\mathbf{B}_{1}$ and $\mathbf{A}_{1}$ are position vectors of $B_{1}$ and $A_{1}$, respectively, and all the vectors are measured in a reference system fixed to the base.

In the $\underline{X} \underline{X} \underline{Y}$ limbs (i.e., the two limb types $\underline{R} \underline{R} \underline{P S}$ and $\underline{P R} \underline{P S}$ ), $\mathbf{A}_{1}, \mathbf{u}_{1}$ and $\mathbf{u}_{2}$ depend only on $\mathrm{x}$; whereas, $\mathrm{y} \equiv \mathrm{d}_{1}$. Therefore, the time derivative of Eq. (B1) is 


$$
\begin{aligned}
\dot{\mathbf{B}}_{1}= & \dot{\mathrm{x}}\left\{\frac{\mathrm{d} \mathbf{A}_{1}}{\mathrm{dx}}+\mathrm{d}_{1}\left[\frac{\mathrm{d} \mathbf{u}_{1}}{\mathrm{dx}} \cos \theta_{2}+\left(\frac{\mathrm{d} \mathbf{u}_{2}}{\mathrm{dx}} \times \mathbf{u}_{1}+\mathbf{u}_{2} \times \frac{\mathrm{d} \mathbf{u}_{1}}{\mathrm{dx}}\right) \sin \theta_{2}\right]\right\}+ \\
& \dot{\mathrm{y}}\left[\mathbf{u}_{1} \cos \theta_{2}+\left(\mathbf{u}_{2} \times \mathbf{u}_{1}\right) \sin \theta_{2}\right]+\mathrm{d}_{1} \dot{\theta}_{2} \mathbf{u}_{2} \times\left[\mathbf{u}_{1} \cos \theta_{2}+\left(\mathbf{u}_{2} \times \mathbf{u}_{1}\right) \sin \theta_{2}\right]
\end{aligned}
$$

whose dot products by $\mathbf{u}_{2}$ and by $\left(\mathbf{B}_{1}-\mathbf{A}_{1}\right)$ yield

$$
\begin{aligned}
& \mathrm{m}_{11}=\mathbf{u}_{2} \cdot\left\{\frac{\mathrm{d} \mathbf{A}_{1}}{\mathrm{dx}}+\mathrm{d}_{1}\left[\frac{\mathrm{d} \mathbf{u}_{1}}{\mathrm{dx}} \cos \theta_{2}+\left(\frac{\mathrm{d} \mathbf{u}_{2}}{\mathrm{dx}} \times \mathbf{u}_{1}\right) \sin \theta_{2}\right]\right\} ; \quad \mathrm{m}_{12}=0 \\
& \mathrm{~m}_{21}=\mathrm{d}_{1}\left[\mathbf{u}_{1} \cos \theta_{2}+\left(\mathbf{u}_{2} \times \mathbf{u}_{1}\right) \sin \theta_{2}\right] \cdot \frac{\mathrm{d} \mathbf{A}_{1}}{\mathrm{dx}} ; \mathrm{m}_{22}=\mathrm{d}_{1}
\end{aligned}
$$

In the XYRS limbs (i.e., the three limb types $\underline{\mathrm{RRRS}} \underline{\mathrm{RPRS}}$ and $\underline{\mathrm{PPRS}}), \mathbf{A}_{1}, \mathbf{u}_{1}$ and $\mathbf{u}_{2}$ depend both on $\mathrm{x}$ and on $\mathrm{y}$. Therefore, the time derivative of Eq. (B1) is

$$
\begin{aligned}
\dot{\mathbf{B}}_{1}= & \dot{\mathrm{x}}\left\{\frac{\partial \mathbf{A}_{1}}{\partial \mathrm{x}}+\mathrm{d}_{1}\left[\frac{\partial \mathbf{u}_{1}}{\partial \mathrm{x}} \cos \theta_{2}+\left(\frac{\partial \mathbf{u}_{2}}{\partial \mathrm{x}} \times \mathbf{u}_{1}+\mathbf{u}_{2} \times \frac{\partial \mathbf{u}_{1}}{\partial \mathrm{x}}\right) \sin \theta_{2}\right]\right\}+ \\
& \dot{\mathrm{y}}\left\{\frac{\partial \mathbf{A}_{1}}{\partial \mathrm{y}}+\mathrm{d}_{1}\left[\frac{\partial \mathbf{u}_{1}}{\partial \mathrm{y}} \cos \theta_{2}+\left(\frac{\partial \mathbf{u}_{2}}{\partial \mathrm{y}} \times \mathbf{u}_{1}+\mathbf{u}_{2} \times \frac{\partial \mathbf{u}_{1}}{\partial \mathrm{y}}\right) \sin \theta_{2}\right]\right\}+\mathrm{d}_{1} \dot{\theta}_{2} \mathbf{u}_{2} \times\left[\mathbf{u}_{1} \cos \theta_{2}+\left(\mathbf{u}_{2} \times \mathbf{u}_{1}\right) \sin \theta_{2}\right]
\end{aligned}
$$

whose dot products by $\mathbf{u}_{2}$ and by $\left(\mathbf{B}_{1}-\mathbf{A}_{1}\right)$ yield

$$
\begin{aligned}
& \mathrm{m}_{11}=\mathbf{u}_{2} \cdot\left\{\frac{\partial \mathbf{A}_{1}}{\partial \mathrm{x}}+\mathrm{d}_{1}\left[\frac{\partial \mathbf{u}_{1}}{\partial \mathrm{x}} \cos \theta_{2}+\left(\frac{\partial \mathbf{u}_{2}}{\partial \mathrm{x}} \times \mathbf{u}_{1}\right) \sin \theta_{2}\right]\right\} \\
& \mathrm{m}_{12}=\mathbf{u}_{2} \cdot\left\{\frac{\partial \mathbf{A}_{1}}{\partial \mathrm{y}}+\mathrm{d}_{1}\left[\frac{\partial \mathbf{u}_{1}}{\partial \mathrm{y}} \cos \theta_{2}+\left(\frac{\partial \mathbf{u}_{2}}{\partial \mathrm{y}} \times \mathbf{u}_{1}\right) \sin \theta_{2}\right]\right\} \\
& \mathrm{m}_{21}=\mathrm{d}_{1}\left[\mathbf{u}_{1} \cos \theta_{2}+\left(\mathbf{u}_{2} \times \mathbf{u}_{1}\right) \sin \theta_{2}\right] \cdot \frac{\partial \mathbf{A}_{1}}{\partial \mathrm{x}} \\
& \mathrm{m}_{22}=\mathrm{d}_{1}\left[\mathbf{u}_{1} \cos \theta_{2}+\left(\mathbf{u}_{2} \times \mathbf{u}_{1}\right) \sin \theta_{2}\right] \cdot \frac{\partial \mathbf{A}_{1}}{\partial \mathrm{y}}
\end{aligned}
$$

In the RXYS limbs (i.e., the two limb types RRPS, RPPS), $\mathbf{u}_{1}$ and $\mathbf{u}_{2}$ are fixed to the base, $\mathbf{A}_{1}$ is constrained to lie on the axis of the passive $\mathrm{R}$ pair so that the relationship $\mathbf{A}_{1}=\mathbf{A}_{10}+\mathrm{x} \mathbf{u}_{2}$ where $\mathbf{A}_{10}$ is a base point can always be introduced together with the position $\mathrm{y} \equiv \mathrm{d}_{1}$. Therefore, the time derivative of Eq. (B1) is 
$\dot{\mathbf{B}}_{1}=\dot{\mathrm{x}} \mathbf{u}_{2}+\dot{\mathrm{y}}\left[\mathbf{u}_{1} \cos \theta_{2}+\left(\mathbf{u}_{2} \times \mathbf{u}_{1}\right) \sin \theta_{2}\right]+\mathrm{d}_{1} \dot{\theta}_{2} \mathbf{u}_{2} \times\left[\mathbf{u}_{1} \cos \theta_{2}+\left(\mathbf{u}_{2} \times \mathbf{u}_{1}\right) \sin \theta_{2}\right]$

whose dot products by $\mathbf{u}_{2}$ and by $\left(\mathbf{B}_{1}-\mathbf{A}_{1}\right)$ yield

$\mathrm{m}_{11}=1 ; \quad \mathrm{m}_{12}=0 ; \quad \mathrm{m}_{21}=0 ; \quad \mathrm{m}_{22}=\mathrm{d}_{1}$

With reference to the US limb of Fig. 4 , if it is generated through a limb of UZZS type (i.e, a UPS limb), point $\mathrm{A}_{2}$ is fixed to the base, $\mathrm{z} \equiv \mathrm{d}_{2}$ and $\dot{\mathbf{B}}_{2} \cdot\left(\mathbf{B}_{2}-\mathbf{A}_{2}\right)=\dot{\mathrm{z}} \mathrm{d}_{2}$ which brings to conclude that $\mathrm{n}=\mathrm{d}_{2}$. Otherwise, that is, if it is generated through a limb of ZUUS type (i.e, either an $\underline{R} U S$ limb or a PUS limb), the position vector $\mathbf{A}_{2}$ depends

only on z, which yields $\dot{\mathbf{A}}_{2}=\dot{\mathrm{z}} \frac{\mathrm{d} \mathbf{A}_{2}}{\mathrm{dz}}$, and $\mathrm{d}_{2}$ is constant, which yields $\dot{\mathbf{B}}_{2} \cdot\left(\mathbf{B}_{2}-\mathbf{A}_{2}\right)=\dot{\mathbf{A}}_{2} \cdot\left(\mathbf{B}_{2}-\mathbf{A}_{2}\right)$; thus $\mathrm{n}=\frac{\mathrm{d} \mathbf{A}_{2}}{\mathrm{dz}} \cdot\left(\mathbf{B}_{2}-\mathbf{A}_{2}\right)$

\section{References}

1. D.L. Pieper, "The Kinematics of Manipulators Under Computer Control," Ph.D. thesis, Stanford University, Stanford (1968).

2. C. Innocenti and V. Parenti-Castelli, "Direct kinematics of the 6-4 fully parallel manipulator with position and orientation uncoupled," Proc. of the European Robotics and Intelligent Systems Conference, Corfou, Greece (1991).

3. F. Pierrot, P. Dauchez, and A. Fournier, "Hexa: a fast six-dof fully parallel robot," Proc. of the 5th International Conference on Advanced Robotics, '91 ICAR, Pisa, Italy (1991), pp. 1158-1163.

4. $\quad$ S.P. Patarinski, and M. Uchiyama, "Position/orientation decoupled parallel manipulator," Proc. of the 7th International Conference on Advanced Robotics, '93 ICAR, Tokyo, Japan (1993), pp. 153-158.

5. J.P. Lallemand, A. Goudali, and S. Zeghloul, "The 6-dof 2-delta parallel robot," ROBOTICA, 15 (4) $407-$ 416 (1997).

6. R. Di Gregorio, "A new decoupled parallel manipulator", Proc. of the 10th International Workshop on Robotics in Alpe-Adria-Danube Region, RAAD 2001, Vienna, Austria (2001). 
7. G. Yang, I.-M. Chen, W. Chen, W. Lin, “ Kinematic design of a six-dof parallel-kinematics machine with decoupled-motion architecture," IEEE Transactions on Robotics 20 (5) 876-884 (2004).

8. Y. Jin, I.-M. Chen, G. Yang, "Kinematic design of a 6-dof parallel manipulator with decoupled translation and rotation," IEEE Transactions on Robotics 22 (3) 545-551 (2006).

9. O. Altuzarra, M. Loizaga, V. Petuya, E. Amezua, "Partially decoupled parallel manipulators based on multiple platforms," Proc. of the 12th IFToMM World Congress on the Theory of Machines and Mechanisms, Besancon, France (2007).

10. S. Briot, V. Arakelian, S. Guégan, "PAMINSA: A new family of partially decoupled parallel manipulators", Mechanism and Machine Theory 44 (2) 425-444 (2009).

11. V. Glazunov, "Design of decoupled parallel manipulators by means of the theory of screws," Mechanism and Machine Theory 45 (2) 239-250 (2010).

12. X. Kong, C.M. Gosselin, "Type synthesis of input-output decoupled parallel manipulators," Trans. Can. Soc. Mech. Eng. 28 (2A) 185-196 (2004).

13. G. Gogu, "Fully-isotropic T3R1-type parallel manipulators, " On Advances in Robot Kinematics, J. Lenarcic and C. Galletti eds., Kluwer Academic Publishers, Dordrecht (Netherlands), pp. 265-272.

14. M. Carricato, "Fully isotropic four-degree-of-freedom parallel mechanisms for Schoenflies motion," Int. J. of Robotics Research 24 (5) 397-414 (2005).

15. X. Kong, C.M. Gosselin, "Type synthesis of 3T1R 4-DOF parallel manipulators based on screw theory," IEEE Trans. on Robotics and Automation 20 (2) 181-190 (2004).

16. P.-L. Richard, C.M. Gosselin, X. Kong, "Kinematic analysis and prototyping of a partially decoupled 4DOF 3T1R parallel manipulator,” ASME J. of Mechanical Design 129 (6) 611-616 (2007).

17. X. Kong, C.M. Gosselin, "Type synthesis of 5-DOF parallel manipulators based on screw theory," J. of Robotic Systems 22 (10) 535-547 (2005).

18. C.M. Gosselin, M. T. Masouleh, V. Duchaine, P.-L. Richard, S. Foucault, X. Kong, "Parallel mechanisms of the multipteron family: kinematic architectures and benchmarking," Proc. of the 2007 IEEE International Conference on Robotics and Automation, ICRA 2007, Rome, Italy (2007), pp. 555-560.

19. I. Zabalza, J. Ros, J.J. Gil, J.M. Pintor, J.M. Jimenez, “TRI-SCOTT. A new kinematic structure for a 6DOF decoupled parallel manipulator," Proc. of the Workshop on Fundamental Issues and Future Research Directions for Parallel Mechanisms and Manipulators, Québec, Canada (2002), pp. 12-15. 
20. Y. Takeda, K. Kamiyama, Y. Maki, M. Higuchi, K. Sugimoto, "Development of position-orientation decoupled spatial in-parallel actuated mechanisms with six degrees of freedom," J. of Robotics and Mechatronics 17 (1) 59-68 (2005).

21. Y. Jin, I.-M. Chen, G. Yang, "Structure synthesis and singularity analysis of a parallel manipulator based on selective actuation," Proc. of the 2004 IEEE International Conference on Robotics and Automation, ICRA 2004, New Orleans, USA (2004), pp. 4533-4538.

22. X. Kong, C.M. Gosselin, "Type synthesis of 6-DOF wrist-partitioned parallel manipulators," ASME Journal of Mechanical Design 130 (6) 062302-(1:8) (2008).

23. G. Legnani, I. Fassi, H. Giberti, S. Cinquemani, D. Tosi, "A new isotropic and decoupled 6-DoF parallel manipulator," Mechanism and Machine Theory 58 (1) 64-81 (2012).

24. X. Kong, C. M. Gosselin, “Type Synthesis of Parallel Mechanisms,'” (Springer-Verlag, Berlin Heidelberg, Germany, 2007) ISBN: 978-3-540-71989-2.

25. C.M. Gosselin, "Determination of workspace of 6-dof parallel manipulators," ASME J. of Mechanical Design, Vol. 112, No. 3, 1990, pp.331-336

26. K. Hunt, “Kinematic Geometry of Mechanisms,” (Clarendon Press, Oxford, UK, 1978)

27. A. Liberati, N.P. Belfiore, "A method for the identification of the connectivity in multi-loop kinematic chains: Analysis of chains with total and partial mobility," Mechanism and Machine Theory 41 (11) 1443 1466 (2006).

28. D. Martins, A. Piga Carboni, "Variety and connectivity in kinematic chains," Mechanism and Machine Theory 43 (10) 1236-1252 (2008).

29. C. Innocenti, V. Parenti-Castelli, "Exhaustive enumeration of fully-parallel kinematic chains," Proc. of the 1994 ASME Int. Winter Annual Meeting, Chicago, USA, DSC-Vol. 55-2, pp. 1135-1141 (1994).

30. M. Takano, "A new effective solution for inverse kinematics problem (synthesis) of a robot with any type of configuration", Journal of the Faculty of Engineering, The University of Tokyo, 38 (2) 107-135 (1985).

31. J. Angeles, "Fundamentals of Robotic Mechanical Systems: Theory, Methods, and Algorithms," $4^{\text {th }}$ Ed., (Springer, New York, 2014)

32. C.M. Gosselin and J. Angeles, "Singularity analysis of closed-loop kinematic chains," IEEE Transactions on Robotics and Automation 6 (3) 281-290 (1990).

33. O. Ma and J. Angeles, "Architecture singularities of platform manipulators," Proc. of the 1991 IEEE Int. Conf. on Robotics and Automation, Sacramento (CA, USA), pp. 1542-1547 (1991). 
34. D. Zlatanov, R.G. Fenton and B. Benhabib, "A unifying framework for classification and interpretation of mechanism singularities," ASME J. of Mechanical Design, 117 (4) 566-572 (1995).

35. L.-W. Tsai, "Robot Analysis: The Mechanics of Serial and Parallel Manipulators," (John Wiley \& Sons, New York (USA), 1999)

36. C.M. Gosselin, "Stiffness mapping for parallel manipulators," IEEE Transactions on Robotics and Automation 6 (3) 377-382 (1990).

37 C.D. Meyer, "Matrix analysis and applied linear algebra," (SIAM, Philadelphia (USA), 2000)

38 J. Angeles, C.S. Lòpez-Cajùn, "Kinematic isotropy and the conditioning index of serial type robotic manipulators," Int. J. of Robotics Research 11 (6) 560-571 (1992). 


\section{Figure Captions}

Figure 1: A structure of type S-RS-US.

Figure 2: Three limbs of (3W)S type: (a) the $\underline{R} R S \operatorname{limb}$, (b) the single-loop $\underline{R} \perp(2 \underline{R} \| R) S$ limb, and (c) singleloop $\underline{\mathrm{R}} \perp(2 \mathrm{R} \underline{\mathrm{P}}) \mathrm{S}$ limb.

Figure 3: The $\underline{R R P S} \underline{R} \underline{R} \underline{P}-U \underline{P S}$ architecture.

Figure 4: S-RS-US structure: notations.

Figure 5: Kinematic schemes of the three limbs of (3W)S type shown in Fig. 2 : (a) scheme of the RRPS limb, (b) scheme of the single-loop $\underline{\mathrm{R}} \perp(2 \underline{\mathrm{R}} \| \mathrm{R}) \mathrm{S}$ limb, and (c) scheme of the single-loop $\underline{\mathrm{R}} \perp(2 \mathrm{R} \underline{\mathrm{P}}) \mathrm{S}$ limb.

Figure 6: Translation singularities: geometric conditions identifying a limb singularity (a) of the single-loop $\underline{\mathrm{R}} \perp(2 \underline{\mathrm{R}} \| \mathrm{R}) \mathrm{S}$ limb and (b) of the single-loop $\underline{\mathrm{R}} \perp(2 \mathrm{R} \underline{\mathrm{P}}) \mathrm{S}$ limb.

Figure 7: Rotation singularities: a configuration that satisfies geometric condition (c).

Figure 8: Studied RRPS-RRPS-UPS architecture: top view of the home position.

Figure 9: Singularity-free workspace of the studied RRPS-RRPS-UPS: top views of the constant-platformorientation configurations where (a) points $A_{0}$ and $O$, (b) points $A_{1}$ and $B_{1}$, and (c) points $A_{2}$ and $B_{2}$ lie on the same vertical line; (d) front view of the configuration (b) with the minimum and maximum limb lengths necessary to make the platform translate of 0.5 l.u. along its normal. 